\title{
High-temperature miscibility of iron and rock during terrestrial planet formation
}

\author{
Sean M.Wahl ${ }^{\mathrm{a},}$, Burkhard Militzer ${ }^{\mathrm{a}, \mathrm{b}}$ \\ ${ }^{a}$ Department of Earth and Planetary Science, University of California Berkeley, United States \\ ${ }^{b}$ Department of Astronomy, University of California Berkeley, United States
}

\begin{abstract}
The accretion of a terrestrial body and differentiation of its silicate/oxide mantle from iron core provide abundant energy for heating its interior to temperatures much higher than the present day Earth. The consequences of differentiation on the structure and composition of planets are typically addressed considering only the interaction of molten iron with an immiscible 'rocky' phase. We demonstrate that mixing in a representative system of liquid or solid $\mathrm{MgO}$ and liquid iron to a single homogeneous liquid occurs at sufficiently low temperature to be present in the aftermath of a giant impact. Applying the thermodynamic integration technique to density functional theory molecular dynamics simulations, we determine the solvus closure temperature for the Fe-MgO system for pressures up to $400 \mathrm{GPa}$. Solvus closure occurs at $\sim 4000 \mathrm{~K}$ at low pressure, and has a weak positive pressure dependence, such that its gradient with respect to depth is less steep than an adiabatic temperature profile. This predicts a new mode of core-mantle differentiation following the most energetic giant impacts, with exsolution of iron from the mixture beginning in the outer layers of the planet. We demonstrate that high-temperature equilibration results in delivery of nominally insoluble $\mathrm{Mg}$-rich material to the early core. Since $\mathrm{MgO}$ is the least soluble major mantle component in iron at low temperatures, these results may represent an upper bound on temperature for mixing in terrestrial planets.
\end{abstract}

Keywords: planet formation, composition, differentiation, miscibility, first principles, iron, $\mathrm{MgO}$, solvus closure

\section{Introduction}

Terrestrial planets are, to first order, made up of a metallic iron core and a mantle composed of silicate and oxide minerals. Chondritic meteorites show that these materials initially condensed together from the protoplanetary nebula, but became free to separate and gravitationally stratify as planetesimals grew. Numerous scenarios have been put forward to describe how these reservoirs interact depending on the pressure, extent of melting, and the specific assumptions of rocky phases (Stevenson, 1990; Solomatov, 2007; Rubie et al., 2007). These typically assume the major components occur in two immiscible phases. Additionally, most studies assume that element partitioning between the two phases is similar to that observed in experiments performed at much lower temperatures (McDonough and Sun, 1995). In the case of a hot early history of a growing planet, neither assumption is necessarily correct. At sufficiently high temperatures, entropic effects dominate and any mixture of materials will form a single, homogeneous phase. It is therefore necessary to consider a high temperature mixture of the 'rocky' and metallic terrestrial components. The presence of such a mixed phase will affect the chemistry of iron-silicate differentiation on the early Earth.

Here we consider a simple representative material for the mixed rock-metal phase as a mixture of $\mathrm{Fe}$ and $\mathrm{MgO}$ formed via the reaction $\mathrm{MgO}_{\text {sol/liq }}+\mathrm{Fe}_{\text {liq }} \Rightarrow \mathrm{FeMgO}_{\text {liq }}$. We determine

\footnotetext{
${ }^{*}$ Corresponding author

Email address: swahl@berkeley.edu (Sean M.Wahl)
}

the stability of these phases using first-principles calculations. At a given pressure, a system with two separate phases can be described in terms of a miscibility gap. At low temperatures, homogeneous mixtures with intermediate compositions are thermodynamically unstable and a heterogeneous mixture of two phases with compositions near the endmembers is preferred. The exsolution gap is bounded by a solvus that marks the temperature above which a single mixed phase is stable, and the maximum temperature along the solvus is referred to as the solvus closure temperature. Here we calculate the Gibbs free energy of the mixture and the endmembers to determine the solvus closure temperature for mixture similar to the bulkcomposition of a terrestrial planet. These results can inform future work, by providing the conditions where rock-metal miscibility plays a role in the differentiation of terrestrial planet interiors.

An order of magnitude calculation shows the gravitational energy released in the formation of an Earth-mass body, if delivered instantaneously, is sufficient to raise temperatures inside the body by $\sim 40,000 \mathrm{~K}$. Redistribution of mass within the body during core formation can account for another $\sim 4,000$ $\mathrm{K}$ increase. This energy is released over the timescale of accretion, $\sim 10^{8}$ years (Chambers and Wetherill, 1998), with efficient surface heat loss through a liquid-atmosphere interface (Abe, 1997; Elkins-Tanton, 2012). However, simulations of the final stages of planet growth (Cameron and Benz, 1991; Chambers and Wetherill, 1998; Canup and Agnor, 2000) suggest that near-instantaneous release of large quantities of energy through giant impacts is the rule rather than the exception. 
Simulations of the 'canonical' moon-forming impact hypothesis (Canup, 2004), in which a Mars-sized body collides with the protoearth, find fractions of the target's interior shocked well above 10,000 K. More recently, dissipation of angular momentum from the Earth-Moon system by the evection resonance has loosened physical constraints on the impact, suggesting that the formation of the moon is better explained by an even more energetic event than the 'canonical' one (Canup, 2012; Cuk and Stewart, 2012). It is, therefore, difficult to precisely constrain the temperature of the Earth's interior in the aftermath of the moon forming impact, much less that of other terrestrial planets with even more uncertain impact histories. Regardless, there is evidence for giant impacts throughout the inner solar system, implying temperatures significantly higher than the present day Earth may have been common. In addition to high temperatures, giant impacts involve significant physical mixing of iron and rocky materials (Dahl and Stevenson, 2010). Thus, miscibility may be important even if the impacting bodies have iron cores that differentiated at lower temperatures and pressures.

Differentiation and core formation is a key event or series of events in terrestrial planet evolution. The timing and conditions of differentiation have important consequences for the evolution of the planet, through its effect on the distribution of elements throughout the planet's interior. The distribution of elements affects the gravitational stability of solid layers in the mantle, the location of radioactive heat sources, and the nature of the source of buoyancy driving core convection and magnetic field generation. Each of these subsequently affect the thermal evolution of the planet. If this process occurs near the solvus closure temperature, there are likely to be physical and chemical differences from the processes at conditions where the phases are completely immiscible. We include a discussion of some of these processes in Section 4.

\section{Theory and methodology}

Modern high pressure experimental techniques, using static or dynamic compression techniques, can reach megabar pressures (Boehler, 2000). However, experiments at simultaneous high pressures and temperatures have limitations. Interpretation of mixing processes during shock wave experiments is difficult, and the samples are not recoverable. Meanwhile, laser heated diamond anvil cells experience extreme temperature gradients and require survival of quenched texture to interpret. In both cases, the methods only cover a small fraction of the $P-T$ range expected in the aftermath of a giant impact. As a result, simulations based on first-principles theories are an appropriate means of constraining material properties over a range of such extreme conditions.

\subsection{Simulated System}

We performed density functional theory molecular dynamics (DFT-MD) simulations for phases in a model reaction between liquid iron, and solid (B1) or liquid magnesium oxide. The change in Gibbs free energy of this system per formula unit $\mathrm{FeMgO}$ is described as

$$
\Delta G_{\text {mix }}=\frac{1}{24} G_{(\mathrm{FeMgO})_{24}}-\frac{1}{32}\left[G_{(\mathrm{MgO})_{32}}+G_{\mathrm{Fe}_{32}}\right]
$$

where $G_{(\mathrm{MgO})_{32}}$ and $G_{\mathrm{Fe}_{32}}$ are the Gibbs free energies of a pure $\mathrm{MgO}$ and iron endmembers with subscripts referring to the number of atoms in the periodic simulation cell. $G_{(\mathrm{FeMgO})_{24}}$ is the Gibbs free energy of 1:1 stoichiometric liquid solution of the two endmembers. Comparing Gibbs free energies among a range of compositions, we find the temperature for mixing of the phases in the 1:1 ratio to be a good approximation for the solvus closure temperature.

$\mathrm{MgO}$ is the simplest mantle phase to simulate, and a reasonable starting point for a study of the miscibility of terrestrial materials. Up to $\sim 400 \mathrm{GPa}, \mathrm{MgO}$ remains in the cubic $\mathrm{B} 1$ $(\mathrm{NaCl})$ structure (Boates and Bonev, 2013), meaning simulations of only one solid phase were necessary for the rocky endmember. In order to describe a similar reaction for $\mathrm{MgSiO}_{3}$ perovskite, the Gibbs free energy of $\mathrm{MgO}$ and $\mathrm{SiO}_{2}$ must also be calculated to address the possibility of incongruent dissolution of the solid phase (Boates and Bonev, 2013). More importantly, high pressure experiments observing reactions of silicates with iron have demonstrated the $\mathrm{MgO}$ component has by far the lowest solubility in iron up to $\sim 3000 \mathrm{~K}$ (Knittle and Jeanloz, 1991; Ozawa et al., 2008). This suggests our results can be interpreted as an upper bound for the solvus closure temperature with more realistic compositions.

It is worth emphasizing that the mixed FeMgO phase is unlike any commonly studied rocky phase, in that it does not have a balanced oxide formula. This is by design and is necessary for the mixing of arbitrary volumes of the metallic and oxide phases. This is a separate process from the reaction of the $\mathrm{FeO}$ component which transfers $\mathrm{O}$ to the metallic phase at lower temperature, and which is primarily controlled by oxygen fugacity rather than temperature (Tsuno et al., 2013). We treat the mixed phase as a liquid at all conditions. Although we cannot absolutely rule out the possibility of a stable solid with intermediate composition, such a phase would require a lower Gibbs free energy, and therefore is consistent with treating our results as an upper bound on the solvus closure temperature.

\subsection{Computation of Gibbs Free Energies}

The Gibbs free energy of a material includes a contribution from entropy of the system. Since entropy is not determined in the standard DFT-MD formalism, we adopt a two step thermodynamic integration method, used in previous studies (Wilson and Militzer, 2010, 2012; Wahl et al., 2013; González-Cataldo et al., 2014). The thermodynamic integration technique considers the change in Helmholtz free energy for a transformation between two systems with governing potentials $U_{a}\left(\mathbf{r}_{\mathbf{i}}\right)$ and $U_{b}\left(\mathbf{r}_{\mathbf{i}}\right)$. We define a hybrid potential $U_{\lambda}=(1-\lambda) U_{a}+\lambda U_{b}$, where $\lambda$ is the fraction of the potential $U_{b}\left(\mathbf{r}_{\mathbf{i}}\right)$. The difference in Helmholtz free energy is then given by

$$
\Delta F_{a \rightarrow b} \equiv F_{b}-F_{a}=\int_{0}^{1} d \lambda\left\langle U_{b}\left(\mathbf{r}_{\mathbf{i}}\right)-U_{a}\left(\mathbf{r}_{\mathbf{i}}\right)\right\rangle_{\lambda}
$$


where the bracketed expression represents the ensembleaverage over configurations, $\mathbf{r}_{\mathbf{i}}$, generated in simulations with the hybrid potential at constant volume and temperature. This technique allows for direct comparisons of the Helmholtz free energy of DFT phases, $F_{\mathrm{DFT}}$, by finding their differences from reference systems with a known analytic expression, $F_{\text {an }}$.

In practice, it is more computationally efficient to perform the calculation $\Delta F_{\text {an } \rightarrow \text { DFT }}$ in two steps, each involving an integral of the form of Eqn. 2. We introduce an intermediate system governed by classical pair potentials, $U_{\mathrm{cl}}$, found by fitting forces to the DFT trajectories (Wilson and Militzer, 2010; Izvekov et al., 2004). For each pair of elements, find the average force in bins of radial separation and fit the shape of a potential using a cubic spline function. We constrain the potential to smoothly approach zero at large radii and use a linear extrapolation at small radii, where the molecular dynamics simulations provide insufficient statistics. Examples of these potentials are included in the online supplementary information. The full energetics of the system is then described as

$$
F_{\mathrm{DFT}}=F_{\mathrm{an}}+\Delta F_{\mathrm{an} \rightarrow \mathrm{cl}}+\Delta F_{\mathrm{cl} \rightarrow \mathrm{DFT}}
$$

where $\Delta F_{\mathrm{cl} \rightarrow \mathrm{DFT}}$ requires a small number of DFT-md simulations, and $\Delta F_{\text {an } \rightarrow \mathrm{cl}}$ numerous, but inexpensive classical Monte Carlo (CMC) simulations. The method depends on a smooth integration of $\Delta F_{\mathrm{cl} \rightarrow \mathrm{DFT}}$ and avoiding any first order phase transitions with $\lambda$. We use five $\lambda$ points, for all $\Delta F_{\mathrm{cl} \rightarrow \text { DFT }}$ integrations. For solid $\mathrm{MgO}$ we use a combination of classical pair and one-body harmonic oscillator potentials for $U_{\mathrm{cl}}$ (Wilson and Militzer, 2012; Wahl et al., 2013). Liquids we use only pair potentials. For solids the analytical reference system is an Einstein solid with atoms in harmonic potentials centered on a perfect lattice, while a gas of non-interacting particles is used for liquids. We found integrating over 5 lambda points to be sufficiently accurate, with an increase to 9 lambda points changing our results by $<0.003 \mathrm{eV}$ per formula unit. In the online supplementary information we include two additional tests of the thermodynamic integration method, demonstrating that our results are not sensitive use of different classical potentials or the integration path with respect to the interaction between different species in the multicomponent systems.

All DFT simulations presented here were performed using the Vienna $a b$ initio simulation package (VASP) (Kresse and Furthmüller, 1996). VASP uses projector augmented wave pseudopotentials (Blochl, 1994) and the exchange-correlation functional of Perdew, Burke and Ernzerhof (Perdew et al., 1996). Although the DFT formalism is based on a zerotemperature theory, DFT-MD simulations at high temperatures have been shown to agree with theory developed for warm dense matter (Driver and Militzer, 2012). We use an iron pseudopotential with valence states described by a $[\mathrm{Mg}] 3 \mathrm{pd}^{6} 4 \mathrm{~s}^{2}$ electron configuration. For consistency, all simulations use Balderesci point sampling, a $600 \mathrm{eV}$ cutoff energy for the plane wave expansion and temperature dependent Fermi-smearing to determine partial orbital occupations. A time step of between 0.5 and $1.0 \mathrm{fs}$ is used depending on the temperature, with the smaller time step used for all simulations with temperatures above $6000 \mathrm{~K}$. We confirmed that the resulting molecular dynamics results are well-converged with respect to the energy cutoff and time step. All presented results involve molecular dynamics simulation lengths of at least 2 ps simulated time at each, with longer simulation times having an insignificant effect on the results of the thermodynamic integration. The largest source of uncertainty was the finite size effect, which we discuss in detail in Section 3.

The workflow for the calculation is as follows: 1) Determine the density of the system at the target pressure and temperature by iteratively correcting the volume until the calculated DFT pressure matches the target pressure with a $1 \%$ tolerance. 2) Perform DFT simulations to determine the internal energy, and fit classical potentials for the thermodynamic integration. 3) Perform the thermodynamic integration with the classical potentials with DFT simulations, 4) and CMC simulations to complete the Helmholtz free energy (Eqn. 3), and by extension the entropy and Gibbs free energy. 5) Calculate the differences in these values at given $P$ and $T$, using Eqn. 1 to determine whether the system mixes or not.

\section{Results}

The online supplementary material includes a table with the results of the calculations for each composition and $P-T$ condition. It includes the density along with the calculated pressure, internal energy, entropy and Gibbs free energy. The stable phase at each condition is determined using Eqn. 1. The point at which the trend in $\Delta G_{\text {mix }}$ at constant $P$ changes sign is the inferred solvus temperature at the 1:1 stoichiometric composition. Fig. 1 shows an example of this trend in $\Delta G$ for the 1:1 mixture at $P=50 \mathrm{GPa}$, along with its components $\Delta U, \Delta P V$ and $-\Delta T S$. Using the convention from Eqn. 1, positive values favor the separation of the material into the endmember phases, while negative values favor the single homogeneous mixed phase. The contributions of the internal energy and volumetric terms are positive, while the entropy provides the negative contribution that promotes mixing at sufficiently high temperature.

Using additional calculations at $P=50 \mathrm{GPa}$, we estimated uncertainties in our calculated Gibbs free energies. The most significant contribution to the uncertainty comes from the finite size of the simulation cells. We estimate the magnitude of this uncertainty by comparing results from larger simulated cells to those of the original system. Fig. 2 compares the values of $\Delta G_{\text {mix }}$ for $\mathrm{Fe}$ and $\mathrm{FeMgO}$ cells with up to twice the number of atoms, and $\mathrm{MgO}$ with up to 100 atoms per cell. From this, we estimate a maximum shift of $<0.1 \mathrm{eV}$ per formula unit. This corresponds to an uncertainty in temperature of $\sim 200 \mathrm{~K}$, roughly an order of magnitude larger than the statistical precision of the calculation. The combined effect of increasing cell size for all systems leads consistently to lower values of $\Delta G_{\text {mix }}$ at both temperatures, and thus, lower predicted solvus closure temperatures. For the subsequent analysis, we consider an estimated uncertainty defined as the largest (positive or negative) shift in the Gibbs free energy for each phase. It should be noted that these estimated error bars can not be strictly viewed as statistical uncertainties, since they are unidirectional and based 


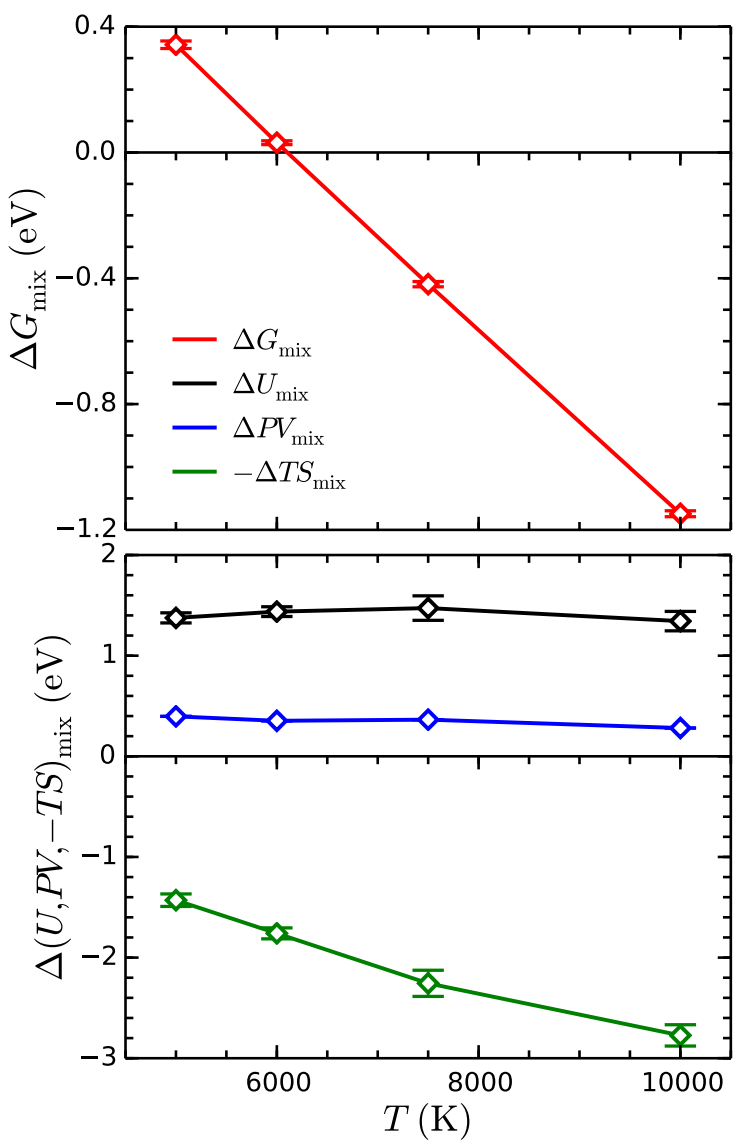

Figure 1. Gibbs free energy change per formula unit, $G_{\text {mix }}$ of the reaction $\mathrm{MgO}_{\text {liq }}+\mathrm{Fe}_{\text {liq }} \rightarrow \mathrm{FeMgO}_{\text {liq }}$ at $P=50 \mathrm{GPa}$ (red). Independently calculated components of $\Delta G_{\text {mix }}: \Delta U$ (black), $\triangle P V$ (blue), and $-\Delta T S$ (green). Positive values favor separation into end member phases, while negative values favor a single mixed phase. $\Delta P V$ values presented here use the target pressure. Error bars represent the integrated error from the $1 \sigma$ statistical uncertainty of the molecular dynamics simulations.

on a small number of independent calculations. They suggest the likely magnitude by which similar shifts in the calculated Gibb's free energies will effect the calculated transition temperature at different $P-T$ conditions.

The effect of pressure and temperature on $\Delta G_{\text {mix }}$ is shown in Fig. 5. As pressure increases, the slope of $\Delta G_{\text {mix }}$ with $T$ remains nearly constant for all simulations with liquid $\mathrm{MgO}$, but the values are shifted to higher temperatures. This means that the solvus closure temperature has a positive slope with pressure over the entire range of conditions considered. There is a noticeable change in the slope of this quantity when $\mathrm{MgO}$ melts. This corresponds with a weaker dependence on pressure at high pressures, where the solvus temperature is below the melting temperature of $\mathrm{MgO}$.

When determining the energetics of the mixed FeMgO, phase

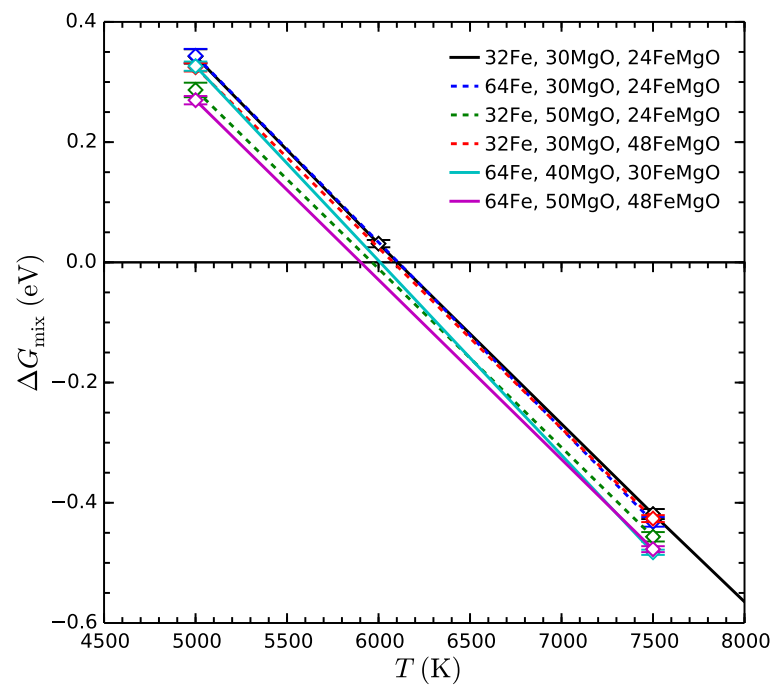

Figure 2. Quantifying the finite size effect on $\Delta G_{\text {mix }}$ for simulations of the reaction $\mathrm{MgO}_{\text {liq }}+\mathrm{Fe}_{\text {liq }} \rightarrow \mathrm{FeMgO}_{\text {liq }}$ at $P=50 \mathrm{GPa}$ with different cell sizes. In black are the results for the systems $\mathrm{Fe}_{32}, \mathrm{Mg}_{32} \mathrm{O}_{32}$ and $\mathrm{Fe}_{24} \mathrm{Mg}_{24} \mathrm{O}_{24}$ used at the other P-T conditions. The other lines show the shift in $\Delta G_{\text {mix }}$ obtained when the calculation is repeated for with a larger cell for one (dashed lines) or all (solid lines) of the systems.

it is important to verify that the simulation remain in a single mixed phase. At temperatures sufficiently close to the solvus closure temperature the system should behave as a super-cooled homogeneous mixture, while at sufficiently low temperatures the simulations could, in principle, spontaneously separate into two phases. This would bias the results as interfacial energies between the separating phases would be included in the calculated Gibbs free energy. We were unable to detect phase separation by visual inspection of various of snapshots as has been seen for hydrogen-helium mixtures in (Soubiran, 2012). The pair correlation function, $g(\mathbf{r})$, can be used as a proxy for separation of phases (Soubiran, 2012).

Fig. 3 shows the Fe-Fe $g(r)$ for simulations of the mixed $\mathrm{FeMgO}$ phase at $50 \mathrm{GPa}$. For temperatures significantly below solvus closure temperature, 3000 and $5000 \mathrm{~K}$, there are slight negative deviations of the $g(r)_{\mathrm{Fe}-\mathrm{Fe}}$ at large $r$ from their expected asymptote to unity. This is consistent with clustering into $\mathrm{MgO}$ and Fe-rich regions, and may indicate spontaneous phase separation at temperatures well below the inferred solvus closure temperature. These deviations are minimal or not observed in simulations near or above the inferred solvus closure temperature. We performed additional simulations at temperatures close to the solvus closure temperature to verify that the Gibbs free energy changes linearly as a function of temperature, which is the expected behaviour without spontaneous phase separation. In spite of deviations in $g(r)$, we note that including the low temperature simulations in our calculation of the solvus closure temperature does not significantly change the result at any pressure.

We also studied the effect of composition on the solvus tem- 


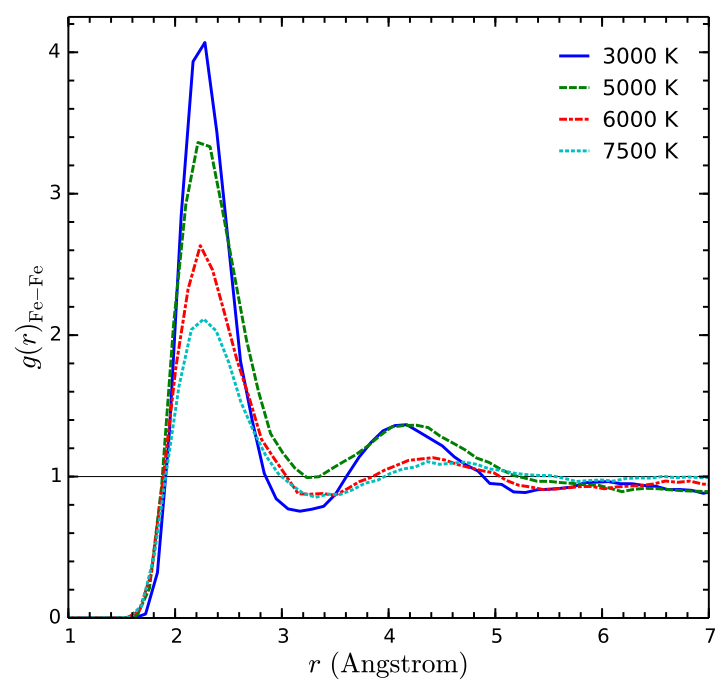

Figure 3. Fe-Fe pair correlation functions for mixed $\mathrm{Fe}+$ $\mathrm{MgO}$ phase. Compares the spatial distribution of atoms in simulations at $50 \mathrm{GPa}$ with different temperatures. The $3000 \mathrm{~K}$ and $5000 \mathrm{~K}$ asymptote to values notably less than one, while temperatures near or above the solvus closure temperature do not show such a deviation at large $r$.

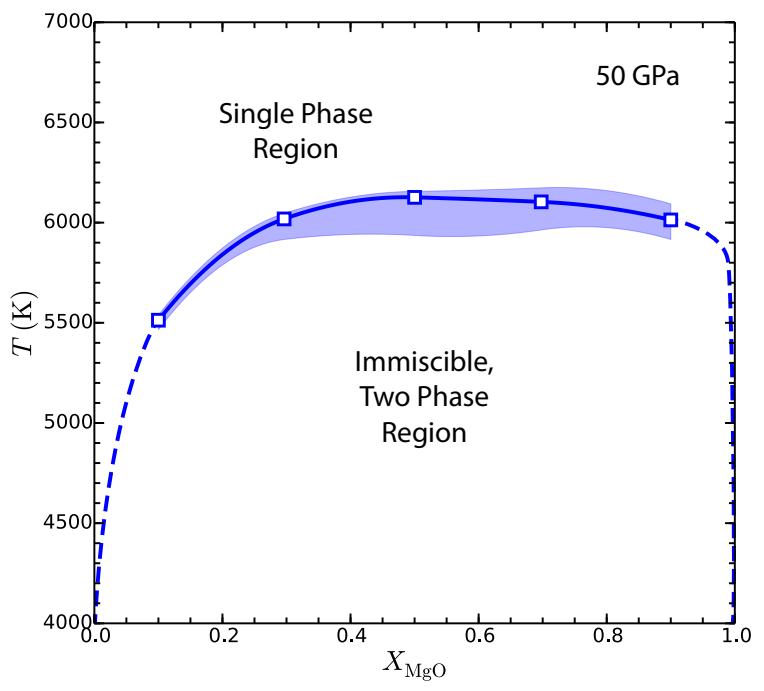

Figure 4. Solvus phase diagram of the $\mathrm{Fe}-\mathrm{MgO}$ system at $P=50 \mathrm{GPa}$. The shape is consistent with the composition $X_{\mathrm{MgO}}=0.5$ being representative for estimating the solvus closure temperature at other pressures. The filled blue region shows an estimate of the uncertainty in transition temperature arising from the uncertainties in $G$ in Fig. 6.

perature. Calculations were performed on four additional intermediate compositions between the $\mathrm{Fe}$ and $\mathrm{MgO}$ endembers. Fig. 6 shows a convex-hull in $G_{\mathrm{Fe}_{1-\mathrm{x}} \mathrm{MgO}_{\mathrm{x}}}$ and $\Delta G_{\text {mix }}$ at $50 \mathrm{GPa}$ and $5000 \mathrm{~K}$. This corresponds to a temperature below the calculated solvus temperature. The Gibbs free energies of all in- termediate components are above the mixing line between the end members, and form a smooth function with composition. This is consistent with a binary system with a miscibility gap. Using linear interpolation between this convex hull and one at $7500 \mathrm{~K}$, we estimate the shape of the miscibility gap at $50 \mathrm{GPa}$, as shown in Fig. 4. The miscibility gap is notably asymmetric, with temperatures decreasing faster towards the Fe-rich endmember than the $\mathrm{MgO}$-rich end. A similar, more pronounced asymmetry has been experimentally determined for the $\mathrm{Fe}-\mathrm{FeO}$ system at lower pressures (Ohtani et al., 1984; Kato and Ringwood, 1989). In spite of this, the shape of the solvus at intermediate compositions, $\sim 0.3-0.9$ molar fraction $\mathrm{MgO}$, is relatively flat. As a result, the temperatures predicted for a 1:1 mixture provide a good estimate for the solvus closure temperature. We note, however, that the shape of miscibility gap may be sensitive to uncertainties from the finite size effect. Considering the estimated errors from the finite size effect test, we can only constrain to be within that $\sim 0.3-0.9 X_{\mathrm{MgO}}$ range. Regardless of this composition our uncertainty in the temperature of solvus closure remains $\sim 200 \mathrm{~K}$.

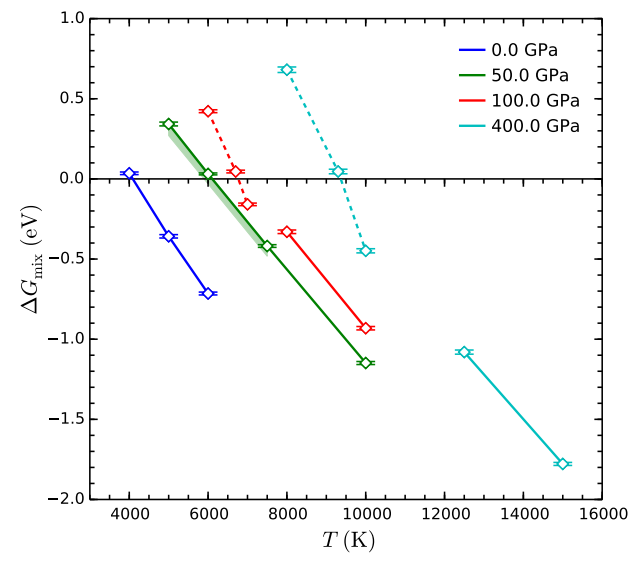

Figure 5. Gibbs free energy of mixing for $\mathrm{MgO}$ and liquid $\mathrm{Fe}$. Solid lines show conditions where $\mathrm{MgO}$ was simulated as a liquid, and dashed lines where $\mathrm{MgO}$ is in its (B1) solid phase. The filled green region shows an estimate of the uncertainty from finite size effects, taken as the maximum shifts in $\Delta G_{\text {mix }}$ observed our tests of larger cells (Fig. 2).

Fig. 7 summarizes the results, showing all the conditions at which simulations were performed. We find the solvus closure at ambient pressure to be $4089_{-235}^{+25} \mathrm{~K}$. While there is little experimental work on this exact system, our results are superficially consistent with extrapolations of the phase diagram for the Fe-FeO system from low temperatures (Ohtani et al., 1984; McCammon et al., 1983), and with the 'accidental' discovery of the Fe-silicate solvus by Walker et al. (1993). We find that the solvus temperature increases with pressure to $6010_{-204}^{+28} \mathrm{~K}$ at 50 $\mathrm{GPa}$, but its slope decreases significantly at higher pressures, with a temperatures of $6767_{-135}^{+14} \mathrm{~K}$ at $100 \mathrm{GPa}$ and $9365_{-130}^{+14}$ $\mathrm{K}$ at $400 \mathrm{GPa}$. This transition also corresponds roughly to the pressure where the trend crosses the $\mathrm{MgO}$ melting curve (Alfè, 2005; Belonoshko et al., 2010; Boates and Bonev, 2013). In- 
deed, the simulations used to infer the closure temperature at these pressures used the solid (B1) structure of MgO. Unfortunately, it is difficult to check whether the change in slope is a direct result of this phase transition, as liquid $\mathrm{MgO}$ simulations rapidly freeze at temperatures far below the melting curve. Conversely, the liquidus of a deep magma ocean might be below the solvus at these temperatures due to the effect of an $\mathrm{SiO}_{2}$ or $\mathrm{FeO}$ component in the silicate/oxide endmember (de Koker et al., 2013; Zerr, 1998). However, extrapolation of $\Delta G_{\text {mix }}$ from simulations with liquid $\mathrm{MgO}$ at higher temperatures (Fig. 5) suggest that the change in slope occurs occurs in liquids as well. We estimate shifts in the inferred solvus closure temperature from finite size effects on the order of $200 \mathrm{~K}$. The actual solvus closure temperature may also be shifted by up to a couple hundred Kelvin, if we also consider the uncertainty in the solvus shape (Fig. 6), since our results refer specifically the solvus temperature for a 1:1 stoichiometric mixture. The observed change in slope of the solvus temperature and the relation to the pure $\mathrm{MgO}$ melting curve are, however, robust against uncertainties of this magnitude.

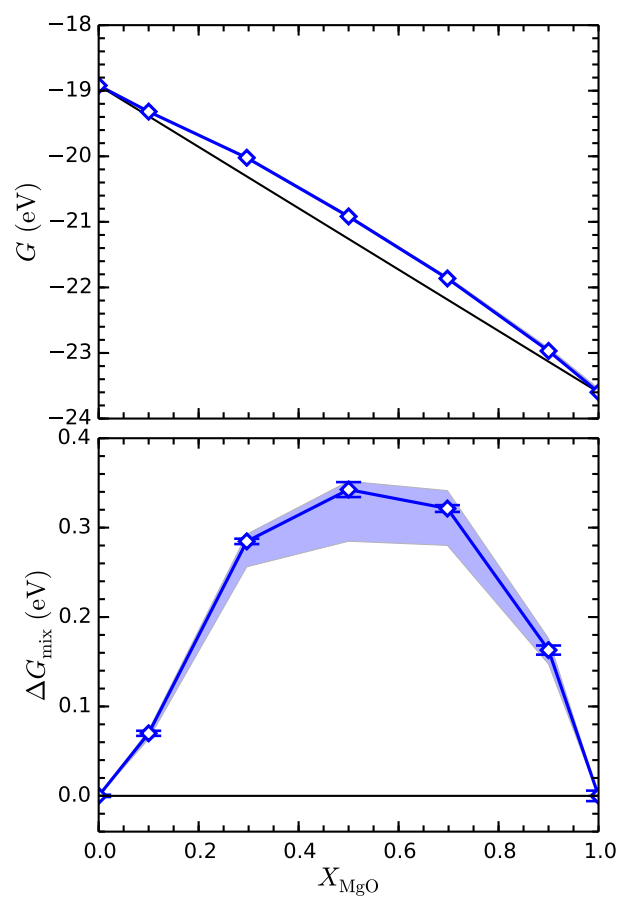

Figure 6. Convex hull of $\Delta G_{\text {mix }}$ versus formula unit fraction, $X_{\mathrm{MgO}}$, for the Fe-MgO system at $P=50 \mathrm{GPa}$ and $T=5000 \mathrm{~K}$ (top). Difference between $\Delta G_{\text {mix }}$ and a mixing line between the endmembers (bottom). The filled blue region shows an estimate of the uncertainty from finite size effects, taken as the maximum shifts in $\Delta G_{\text {mix }}$ from our tests of larger Fe, $\mathrm{MgO}$ and FeMgO simulations. The estimated error is weighted as a function of composition since the finite size effects will cancel with that of the end-member as the compositions become more similar.

\section{Discussion}

In the extreme case where a significant fraction of the planet is in a mixed iron-rock phase, the early evolution will be quite different than prevailing theories. Differentiation of material accreted onto the planet is delayed until the planet cools to below the solvus closure temperature, allowing iron to exsolve and sink to the core. This study provides an estimate of the temperatures required to mix the $\mathrm{Mg}$-rich rocky mantle with the core of a terrestrial planet. At the surface, the complete mixing of $\mathrm{Fe}$ and $\mathrm{MgO}$ is achieved at $\sim 4000 \mathrm{~K}$ (Fig. 7), which is well above the melting point of silicates. At core-mantle boundary pressures, the critical temperature would be $\sim 7000 \mathrm{~K}$. This is below higher estimates for the melting temperature of pure MgO (Alfè, 2005; Belonoshko et al., 2010; Boates and Bonev, 2013) and $\mathrm{MgSiO}_{3}$ perovskite (Zerr and Boehier, 1993). There are significant disparities among calculations and experiments on the melting (Belonoshko, 1994; Belonoshko and Dubrovinsky, 1997; Alfè, 2005; Zerr, 1998) temperatures in the lower mantle, disagreeing even on which phases represent the solidus and liquidus. The melting behavior in our $\mathrm{MgO}$ simulations are consistent with the high-temperature melting curve of recent first-principles simulations (Alfè, 2005; Belonoshko et al., 2010; Boates and Bonev, 2013). Regardless, the solvus remains well above the solidus for more realistic compositions of the silicate mantle (de Koker et al., 2013; Zerr, 1998; Holland, 1997).

\subsection{Evolution of a fully mixed planet}

For a sufficiently energetic impact, or series of impacts, a planet might be heated to such high temperatures, that the entire planet maybe be an approximately homogeneous mixture of the iron and rock components. Such an extreme scenario is unlikely for an Earth-sized planet, and likely violates geochemical observations that preclude complete mixing of the Earth's primitive mantle (Mukhopadhyay, 2012). Nonetheless, considering the evolution of a planet from a fully-mixed state is useful for demonstrating the effects our phase diagram on the mixing behavior in a planet. A fully mixed state is also not so far-fetched for super-Earths since heating from release of gravitational energy scales as roughly $M^{2 / 3}$.

The depth at which the phases separate from the fully mixed state is determined by the pressure dependence of solvus closure. Following such a large impact, the planet will quickly evolve to a magma ocean state, and a higher-temperature adiabat will be rapidly re-established. Fig. 8 compares the solvus closure temperature to the calculated isentropes of the mixed $\mathrm{FeMgO}$ phase. For a homogeneous, vigorously convecting liquid layer of the planet, these approximate adiabatic temperature profiles of the interior of the planet at different points in its evolution. The comparison is qualitatively the same if the isentropes for either endmember is used instead of the mixed phase. At pressures above $50 \mathrm{GPa}$, the isentropes have a notably steeper slope than the solvus closure temperature. At lower pressures, $<50 \mathrm{GPa}$ the slopes are identical within the estimated uncertainty. As a result, separation begins in the exterior of the planet and proceeds inwards as the planet cools. Since iron separating in the outer portion of the planet is denser than the rocky 


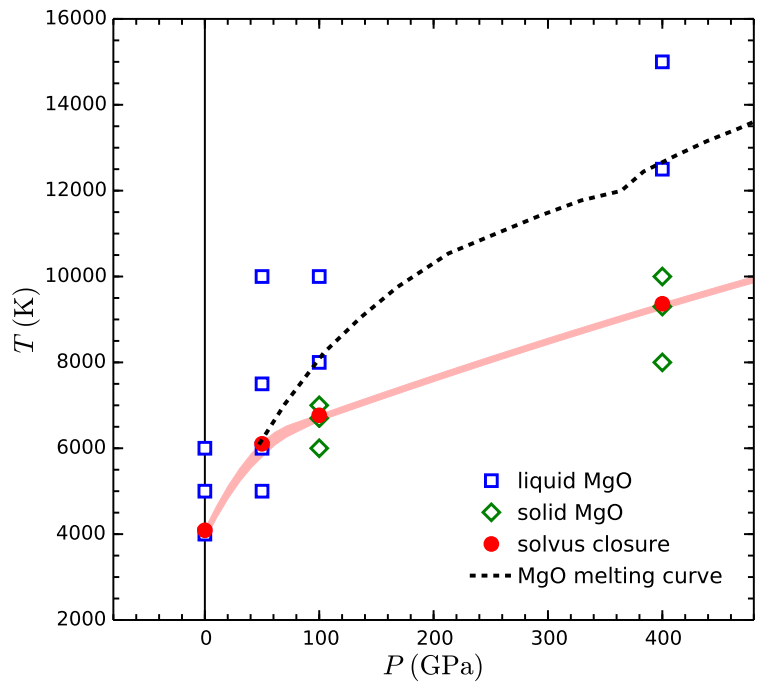

Figure 7. Pressure dependence of the solvus closure. The $P-T$ condition of all thermodynamic integration calculations are included. Blue markers denote conditions where $\mathrm{MgO}$ was treated as a liquid. Green markers denote conditions where $\mathrm{MgO}$ was treated as a solid (B1). Red circles show the solvus closure temperature inferred from simulations at the same pressure. The estimated uncertainty in the solvus closure temperature from finite size effects is shown by the filled red region. The dashed, black line shows the $\mathrm{MgO}$ melting temperature from molecular dynamics from DFT-md with PBE exchange correlation function(Boates and Bonev, 2013), which is consistent with other first-principles calculations (Alfè, 2005; Belonoshko et al., 2010)

phase, it would sink until it reached a depth where it dissolves into the mixed phase again. This may promote compositional stratification, and possibly multi-layer convection between an upper iron-poor and deeper iron-rich layer. The extent to which this process can stratify the planet depends on the competition between growth of liquid Fe droplets and their entrainment in convective flows (Solomatov, 2007).

Based on the Fe-MgO solvus closure temperature presented here, transition of a planet from a fully mixed state to separated rocky and metallic phases would occur while the entire planet is at least partially molten. Consequently, a fully mixed state in an Earth-mass planet would be short lived, since cooling timescales for a deep magma ocean are short in comparison to the timescale of accretion (Abe, 1997; Elkins-Tanton, 2012). This also means little record of such an event is likely to survive to the present day Earth. Indeed, there is little unambiguous evidence for a magma ocean, despite it being a seemingly unavoidable consequence of the moon-forming impact hypothesis. The high surface temperatures of some rocky exoplanets (Pepe et al., 2013) might allow for prolonged cooling times from a such a mixed state.

At the relatively low pressures of growing planetesimals (Kleine et al., 2002; Šrámek et al., 2012; Weiss and ElkinsTanton, 2013), these results predict that core formation begins at temperatures well below the solvus. As a result, complete mixing of a planet must overcome the gravitationally stable differentiated structure. This will impede upward mixing of a dense core even at temperatures above solvus closure, leading to an inefficient double-diffusive convection state like that proposed for the giant planets (Chabrier and Baraffe, 2007). Material accreted while the planet was above the solvus would, however, remain in a fully mixed outer layer, and evolve according to the picture presented in Fig. 8. Substantial mechanical mixing during giant impact events (Canup, 2004, 2012; Cuk and Stewart, 2012) would also enhance mixing prior to the setup of a double-diffusive state.

\subsection{Consequences of localized heating}

Despite the implausibility of a fully-mixed earth, related processes may become important as material is added by impacts with smaller differentiated bodies at temperatures near or above solvus closure. Since peak shock temperatures are related to the velocity of the impact rather than the size of the impactor, smaller-scale events can create localized regions where the temperature exceeds the solvus closure temperature. Assuming iron from the shocked region can be rapidly delivered to the core without significant cooling (Monteux et al., 2009), material equilibrated near or above the solvus can be delivered to the core, through a mantle of lower average temperature. In the case that heat transfer from the sinking iron diapir is negligible, the comparison between the solvus closure temperature and adiabats is valid for the fraction of the planet in contact with the sinking iron. In other words, the temperature in the sinking iron will follow a nearly adiabatic path, with $\mathrm{Fe}$ and $\mathrm{MgO}$ becoming more soluble as the pressure rises. This means that a fraction of the iron delivered to the core could have equilibrated with the rocky mantle at much higher temperatures than on average. The differentiation of a fraction of the planet in the presence of a mixed phase would likely effect partitioning of both major and minor elements between the core and mantle. Walker et al. (1993) suggested that deviations in siderophile element partitioning behavior occur near the solvus closure temperature for iron-silicate mixtures. However, this interpretation has been questioned in light of the confounding effect of drastic changes in oxygen partitioning with pressure (Frost et al., 2010). Better characterization of element-partitioning at such high temperatures could constrain what fraction of the mantle could have been equilibrated in this fashion.

One important consequence of high-temperature equilibration is the delivery of excess, nominally insoluble, light components to the core. This will occur if iron is equilibrated with rocky materials at near-solvus temperatures, and rapidly delivered to the core before it can cool and re-equilibrate with the mantle at lower temperatures. This would be followed by exsolution of a Mg-rich material at the top of the cooling core. This process has been suggested as a possible solution to the problem of the Earth's core having insufficient energy to generate a magnetic field before nucleation of the inner core (Stevenson, 2012). If the interpretation of Fig. 8 can be extended to more iron rich compositions, then exsolution will occur at the top of the core, depositing sediments of Mg-rich material at the 


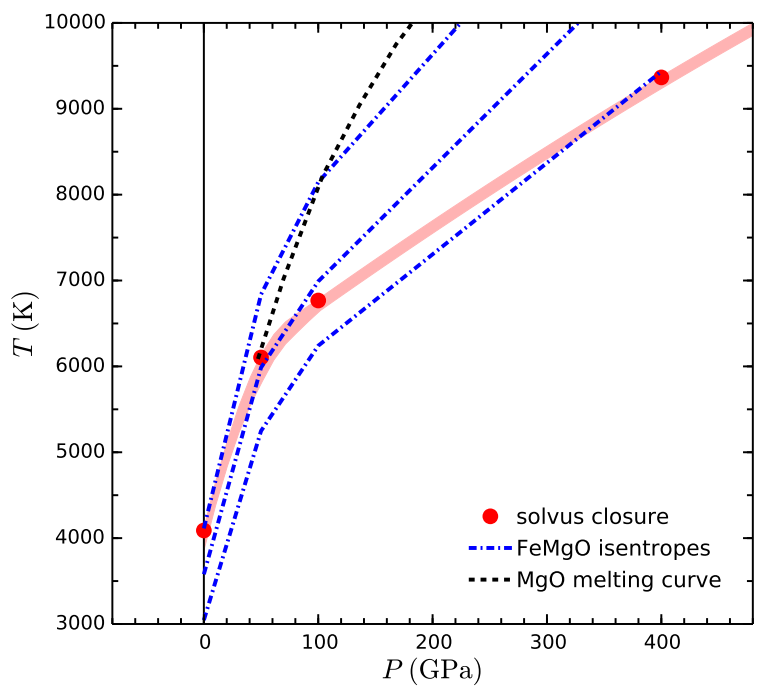

Figure 8. Calculated isentropes for the mixed FeMgO liquid phase compared to the solvus closure temperature. These results favor the mixed phase to remain stable at depth. The dashed, black line shows the $\mathrm{MgO}$ melting temperature from (Boates and Bonev, 2013). The filled regions represent the propagation of estimated errors from finite size effects.

core-mantle boundary (Buffett, 2000). As a result, the effect of this sedimentation on core convection is analogous to the exclusion of light elements from the growing inner core. Fig. 9 shows an extrapolation of our results to predict the saturation of $\mathrm{MgO}$ in $\mathrm{Fe}$ at $50 \mathrm{GPa}$ as a function of temperature. This is done using a function for $G$ in terms of the cell volumes derived in the low-concentration limit (Wilson and Militzer, 2012; Wahl et al., 2013), and details are presented in the supplemental material. From this we predict a $>1 \% \mathrm{MgO}$ saturation limit down to $4200 \mathrm{~K}$, with concentrations steeply decreasing to be below detection limits in high-pressure experiments by $\sim 3000$ K (Knittle and Jeanloz, 1991; Ozawa et al., 2008). In principle, high-temperature equilibration could also explain a bulk-mantle iron concentration in disequilibrium with the present-day core (Ozawa et al., 2008; Tsuno et al., 2013). However, the shape of the $\mathrm{MgO}$-rich side of the calculated exsolution gap (Fig. 4) contradicts this, since the $\mathrm{Fe}$ content of the $\mathrm{MgO}$ endmember shows a significant deviation for only a small range of temperatures.

\section{Conclusions}

The solvus closure temperatures for material with the bulk terrestrial planet composition marks the transition to a regime where where miscibility is a dominant effect in the evolution of the planet. These results present an estimate of those temperatures based on the simplified Fe-MgO system. Where possible, our simulated system was chosen to provide an upper limit for these temperatures, so we expect miscibility for realistic terrestrial compositions at possibly lower temperatures than those found here. The solvus closure temperature found here

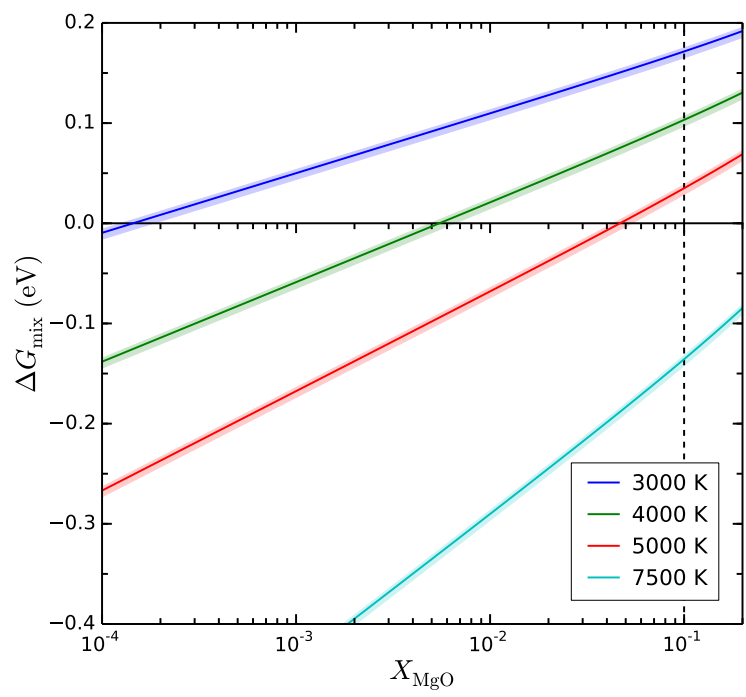

Figure 9. Extrapolated saturation limits of $\mathrm{MgO}$ in $\mathrm{Fe}$ at 50 $\mathrm{GPa}$ at various temperatures. Extrapolation is under the assumption that the solution behaves in the low-concentration limit. The dashed vertical line is the most Fe-rich composition from Fig. 6, from which the extrapolation is made. The filled regions represent the propagation of estimated errors from finite size effects.

for the $\mathrm{Fe}-\mathrm{MgO}$ system is at temperatures low enough, that it was likely overcome for some fraction of the planet during accretion. Energetic impact events are now thought to have been commonplace during the formation of the terrestrial planets, and the role of miscibility between the most abundant rocky and metallic materials should be considered to adequately assess their early evolution.

\section{Acknowledgements}

This work was supported by NASA and NSF. Computational resources were provided in part by the NASA Advanced Supercomputing Division. We thank Dave Stevenson, Raymond Jeanloz, Sarah Stewart and James Brenan for helpful discussions.

\section{References}

\section{References}

Abe, Y., Mar. 1997. Thermal and chemical evolution of the terrestrial magma ocean. Phys. Earth Planet. Inter. 100 (1-4), 27-39.

URL http://linkinghub.elsevier.com/retrieve/pii/ S0031920196032293

Alfè, D., Jun. 2005. Melting Curve of MgO from First-Principles Simulations. Phys. Rev. Lett. 94 (23), 4-7.

URL http://link.aps.org/doi/10.1103/PhysRevLett.94. 235701

Belonoshko, A., 1994. Molecular dynamics of $\mathrm{MgSiO} 3$ perovskite at high pressures : Equation of state, structure, and melting transition. Geochim. Cosmochim. Acta 58 (19), 4039-4047.

URL http://www.sciencedirect.com/science/article/pii/ 0016703794902658 
Belonoshko, A., Dubrovinsky, L., 1997. simulation study of induced failure and recrystallization of a perfect $\mathrm{MgO}$ crystal under non-hydrostatic compression: Application to melting in the diamond-anvil. Am. Mineral. 82, 441-451.

Belonoshko, a. B., Arapan, S., Martonak, R., Rosengren, a., Feb. 2010. MgO phase diagram from first principles in a wide pressure-temperature range. Phys. Rev. B 81 (5), 054110.

URL http://link.aps.org/doi/10.1103/PhysRevB.81.054110

Blochl, P. E., 1994. Projector augmented-wave method. Phys. Rev. B 50 (24), 17953-78.

Boates, B., Bonev, S. A., Mar. 2013. Demixing Instability in Dense Molten $\mathrm{MgSiO}_{3}$ and the Phase Diagram of MgO. Phys. Rev. Lett. 110 (13), 135504. URL http://link.aps.org/doi/10.1103/PhysRevLett.110. 135504

Boehler, R., 2000. High-pressure experiments and the phase diagram of lower mantle and core materials. Rev. Geophys. 38 (2), 221-245. URL http://onlinelibrary.wiley.com/doi/10.1029/ 1998RG000053/full

Buffett, B. A., Nov. 2000. Sediments at the Top of Earth's Core. Science 290 (5495), 1338-1342.

URL http://www.sciencemag.org/cgi/doi/10.1126/science. 290.5495 .1338

Cameron, A. G., Benz, W., Jan. 1991. The origin of the moon and the singleimpact hypothesis IV. Icarus 92, 204-16. URL http://www.ncbi.nlm.nih.gov/pubmed/11542164

Canup, R., Agnor, C., 2000. Accretion of the terrestrial planets and the Earth-Moon system. In: Canup, R. M., Righter, K. (Eds.), Orig. Earth Moon. University of Arizona Press, pp. 113-130.

URL http://books.google.com/books?hl=en\&lr=\&id= $8 i 44 z$ jcKm4EC\&o $i=$ fnd\&pg=PA113\&dq=Accretion+of + the + Terrestrial+Planets+and+the+Earth-Moon+System\&ots= 7J5zasN-sN\&sig=2uIoI6_S3wZVJVDU73YILvAHQx4

Canup, R. M., Sep. 2004. Dynamics of Lunar Formation. Annu. Rev. Astron. Astrophys. 42 (1), 441-475.

URL http://www. annualreviews.org/doi/abs/10.1146/ annurev.astro.41.082201.113457

Canup, R. M., Oct. 2012. Forming a Moon with an Earth-Like Composition via a Giant Impact. Science 1052.

URL http://www.ncbi.nlm.nih.gov/pubmed/23076098

Chabrier, G., Baraffe, I., 2007. Heat transport in giant (exo)planets: A new perspective. Astrophys. J. Lett. 661 (1), L81-L84.

URL http: //iopscience.iop.org/1538-4357/661/1/L81

Chambers, J., Wetherill, G., 1998. Making the terrestrial planets: N-body integrations of planetary embryos in three dimensions. Icarus 327, 304-327. URL http://www.sciencedirect.com/science/article/pii/ S0019103598960079

Cuk, M., Stewart, S. T., Oct. 2012. Making the Moon from a Fast-Spinning Earth: A Giant Impact Followed by Resonant Despinning. Science 1047. URL http://www.ncbi.nlm.nih.gov/pubmed/23076099

Dahl, T. W., Stevenson, D. J., Jun. 2010. Turbulent mixing of metal and silicate during planet accretion - And interpretation of the Hf-W chronometer. Earth Planet. Sci. Lett. 295 (1-2), 177-186.

URL http://linkinghub.elsevier.com/retrieve/pii/ S0012821X10002220

de Koker, N., Karki, B. B., Stixrude, L., Jan. 2013. Thermodynamics of the $\mathrm{MgO}-\mathrm{SiO}_{2}$ liquid system in Earth's lowermost mantle from first principles. Earth Planet. Sci. Lett. 361, 58-63.

URL http://linkinghub.elsevier.com/retrieve/pii/ S0012821X12006449

Driver, K. P., Militzer, B., Mar. 2012. All-Electron Path Integral Monte Carlo Simulations of Warm Dense Matter: Application to Water and Carbon Plasmas. Phys. Rev. Lett. 108 (11), 115502.

URL http://link.aps.org/doi/10.1103/PhysRevLett.108. 115502

Elkins-Tanton, L. T., May 2012. Magma Oceans in the Inner Solar System. Annu. Rev. Earth Planet. Sci. 40 (1), 113-139.

URL http://www.annualreviews.org/doi/abs/10.1146/ annurev-earth-042711-105503

Frost, D. J., Asahara, Y., Rubie, D. C., Miyajima, N., Dubrovinsky, L. S., Holzapfel, C., Ohtani, E., Miyahara, M., Sakai, T., Feb. 2010. Partitioning of oxygen between the Earth's mantle and core. J. Geophys. Res. 115 (B2),
$1-14$.

URL http://www.agu.org/pubs/crossref/2010/2009JB006302. shtml

González-Cataldo, F., Wilson, H. F., Militzer, B., May 2014. Ab Initio Free Energy Calculations of the Solubility of Silica in Metallic Hydrogen and Application To Giant Planet Cores. Astrophys. J. 787 (1), 79.

URL http://stacks.iop.org/0004-637X/787/i=1/a=79?key= crossref .8ed04244b9a9a55eef be9b400819222b

Holland, K. G., Mar. 1997. Melting of $(\mathrm{Mg}, \mathrm{Fe})_{2} \mathrm{SiO}_{4}$ at the Core-Mantle Boundary of the Earth. Science 275 (5306), 1623-1625.

URL http://www.sciencemag.org/cgi/doi/10.1126/science. 275.5306. 1623

Izvekov, S., Parrinello, M., Burnham, C. J., Voth, G. a., Jun. 2004. Effective force fields for condensed phase systems from ab initio molecular dynamics simulation: a new method for force-matching. J. Chem. Phys. 120 (23), 10896-913.

URL http: //www.ncbi.nlm.nih.gov/pubmed/15268120

Kato, T., Ringwood, A. E., Jul. 1989. Melting relationships in the system Fe$\mathrm{FeO}$ at high pressures: Implications for the composition and formation of the earth's core. Phys. Chem. Miner. 16 (6), 524-538.

URL http: //link. springer . com/10.1007/BF00202207

Kleine, T., Münker, C., Mezger, K., Palme, H., Aug. 2002. Rapid accretion and early core formation on asteroids and the terrestrial planets from $\mathrm{Hf}-\mathrm{W}$ chronometry. Nature 418 (6901), 952-5. URL http://www.ncbi.nlm.nih.gov/pubmed/12198541

Knittle, E., Jeanloz, R., 1991. Earth's Core-Mantle Boundary: Results of Experiments at High Pressures and Temperatures. Science 251 (5000), 1438-1443.

URL http://www.sciencemag.org/content/251/5000/1438. short

Kresse, G., Furthmüller, J., Oct. 1996. Efficient iterative schemes for ab initio total-energy calculations using a plane-wave basis set. Phys. Rev. B-Cond. Mat. 54 (16), 11169-11186.

URL http://www.ncbi.nlm.nih.gov/pubmed/9984901

McCammon, C. A., Ringwood, A. E., Jackson, I., 1983. Thermodynamics of the system $\mathrm{Fe}-\mathrm{FeO}-\mathrm{MgO}$ at high pressure and temperature and a model for formation of the Earth's core. Geophys. J. R. Astron. Assoc. 72, 577-95. URL http://onlinelibrary.wiley.com/doi/10.1111/j. 1365-246X.1983.tb02821.x/abstract

McDonough, W., Sun, S., 1995. The composition of the Earth. Chem. Geol. 2541 (94).

URL http://www.sciencedirect.com/science/article/pii/ 0009254194001404

Monteux, J., Ricard, Y., Coltice, N., Dubuffet, F., Ulvrova, M., Oct. 2009. A model of metal-silicate separation on growing planets. Earth Planet. Sci. Lett. 287 (3-4), 353-362.

URL http://linkinghub.elsevier.com/retrieve/pii/ S0012821X09004890

Mukhopadhyay, S., Jun. 2012. Early differentiation and volatile accretion recorded in deep-mantle neon and xenon. Nature 486 (7401), 101-4. URL http://www.ncbi.nlm.nih.gov/pubmed/22678288

Ohtani, E., Ringwood, A. E., Hibberson, W., Nov. 1984. Composition of the core, II. Effect of high pressure on solubility of $\mathrm{FeO}$ in molten iron. Earth Planet. Sci. Lett. 71 (1), 94-103.

URL http://linkinghub.elsevier.com/retrieve/pii/ $0012821 \times 84900554$

Ozawa, H., Hirose, K., Mitome, M., Bando, Y., Sata, N., Ohishi, Y., Mar. 2008 Chemical equilibrium between ferropericlase and molten iron to $134 \mathrm{GPa}$ and implications for iron content at the bottom of the mantle. Geophys. Res. Lett. 35 (5), L05308.

URL http://doi .wiley.com/10.1029/2007GL032648

Pepe, F., Cameron, A. C., Latham, D. W., Molinari, E., Udry, S., Bonomo, A. S., Buchhave, L. A., Charbonneau, D., Cosentino, R., Dressing, C. D., Dumusque, X., Figueira, P., Fiorenzano, A. F. M., Gettel, S., Harutyunyan, A., Haywood, R. D., Horne, K., Lopez-Morales, M., Lovis, C., Malavolta, L., Mayor, M., Micela, G., Motalebi, F., Nascimbeni, V., Phillips, D., Piotto G., Pollacco, D., Queloz, D., Rice, K., Sasselov, D., Ségransan, D., Sozzetti, A., Szentgyorgyi, A., Watson, C. A., Nov. 2013. An Earth-sized planet with an Earth-like density. Nature 503 (7476), 377-80.

URL http: //www.ncbi.nlm.nih.gov/pubmed/24172902

Perdew, J. P., Burke, K., Ernzerhof, M., Oct. 1996. Generalized Gradient Ap- 
proximation Made Simple. Phys. Rev. Lett. 77 (18), 3865-3868

URL http://www.ncbi.nlm.nih.gov/pubmed/10062328

Rubie, D. C., Nimmo, F., Melosh, H. J., 2007. Formation of Earths core. Treatise Geophys. 9, 51-90.

Solomatov, V., 2007. Magma oceans and primordial mantle differentiation. In: Treatise Geophys. Vol. 9. pp. 1-75.

URL ftp://ftp.gps.caltech.edu/pub/djs/solomatov/ Solomatov.pdf

Soubiran, F., 2012. Physique des plasmas denses: le mélange hydrogènehélium dans les intérieurs planétaires. Ph.D. thesis, Centre de Recherche Astrophysique de Lyon.

URL http://hal .archives-ouvertes.fr/tel-00760002/

Stevenson, D., 2012. How to keep a Dynamo Running in spite of High Thermal Conductivity. AGU Fall Meet. Abstr.

URL http: //adsabs . harvard . edu/abs/2012AGUFMDI11C. .03S

Stevenson, D. J., 1990. Fluid dynamics of core formation. Oxford University Press, New York, pp. 231-249.

Tsuno, K., Frost, D. J., Rubie, D. C., Jan. 2013. Simultaneous partitioning of silicon and oxygen into the Earth's core during early Earth differentiation. Geophys. Res. Lett. 40 (1), 66-71.

URL http://doi.wiley.com/10.1029/2012GL054116

Šrámek, O., Milelli, L., Ricard, Y., Labrosse, S., Jan. 2012. Thermal evolution and differentiation of planetesimals and planetary embryos. Icarus 217 (1), 339-354.

URL http://linkinghub.elsevier.com/retrieve/pii/ S0019103511004489

Wahl, S. M., Wilson, H. F., Militzer, B., Aug. 2013. Solubility of Iron in Metallic Hydrogen and Stability of Dense Cores in Giant Planets. Astrophys. J. 773 (2), 95.

URL http://stacks.iop.org/0004-637X/773/i=2/a=95?key= crossref . eb72013b406f986f $46118584 f 8 f 7 a 541$

Walker, D., Norby, L., Jones, J. H., Dec. 1993. Superheating effects on metalsilicate partitioning of siderophile elements. Science 262 (5141), 1858-61. URL http://www.ncbi.nlm.nih.gov/pubmed/17829633

Weiss, B. P., Elkins-Tanton, L. T., May 2013. Differentiated Planetesimals and the Parent Bodies of Chondrites. Annu. Rev. Earth Planet. Sci. 41 (1), 529-560.

URL http://www. annualreviews.org/doi/abs/10.1146/ annurev-earth-040610-133520

Wilson, H. F., Militzer, B., Mar. 2010. Sequestration of noble gases in giant planet interiors. Phys. Rev. Lett. 104 (12), 121101.

URL http: //www.ncbi.nlm.nih.gov/pubmed/20366523

Wilson, H. F., Militzer, B., Mar. 2012. Rocky Core Solubility in Jupiter and Giant Exoplanets. Phys. Rev. Lett. 108 (11), 111101.

URL http://link.aps.org/doi/10.1103/PhysRevLett.108. 111101

Zerr, A., Jul. 1998. Solidus of Earth's Deep Mantle. Science 281 (5374), 243-246.

URL http://www.sciencemag.org/cgi/doi/10.1126/science. 281.5374 .243

Zerr, A., Boehier, R., Oct. 1993. Melting of (Mg,Fe)SiO 3 -Perovskite to 625 Kilobars: Indication of a High Melting Temperature in the Lower Mantle. Science 262 (5133), 553-555.

URL http://www.sciencemag.org/content/262/5133/553.

abstract 


\title{
High-temperature miscibility of iron and rock during terrestrial planet formation
}

\author{
Sean M.Wahl ${ }^{\mathrm{a},}$, Burkhard Militzer ${ }^{\mathrm{a}, \mathrm{b}}$ \\ ${ }^{a}$ Department of Earth and Planetary Science, University of California Berkeley, United \\ States \\ ${ }^{b}$ Department of Astronomy, University of California Berkeley, United States
}

\section{Supplementary material}

\subsection{Simulation results}

Table 1 shows the results of thermodynamic integration calculations performed for each composition and $P-T$ condition. It includes the density along with the calculated pressure, internal energy $U$, entropy $S$, and Gibbs free energy, $G$. Calculations are specified by pressure, $P$, temperature, $T$, and the atomic composition and phase.

To determine the density $\rho$ for a given $P$ and $T$ of interest, we fitted equations of state to results from DFT-MD simulations. $P$ and $U$ are timeaveraged results from DFT-MD simulations with $1 \sigma$ statistical error quoted. $S$ and $G$ are calculated from the two step thermodynamic integration technique. For quantities calculated using the thermodynamic integration, the quoted errors were derived by propagating the errors from each integration point.

Simulations of $\mathrm{Fe}_{32}, \mathrm{Mg}_{32} \mathrm{O}_{32}$ and $\mathrm{Mg}_{24} \mathrm{Fe}_{24} \mathrm{O}_{24}$ are included for every $P-T$ condition. Additional compositions $\left(\mathrm{Mg}_{36} \mathrm{Fe}_{4} \mathrm{O}_{36}, \mathrm{Mg}_{30} \mathrm{Fe}_{13} \mathrm{O}_{30}, \mathrm{Mg}_{16} \mathrm{Fe}_{38} \mathrm{O}_{16}\right.$ and $\mathrm{Mg}_{6} \mathrm{Fe}_{54} \mathrm{O}_{6}$ ) were performed for $50 \mathrm{GPa}$ at 5000 and $7500 \mathrm{~K}$, to test the compositional dependence of the Fe-MgO solvus. Finally, simulations with 1:1 stoichiometries but larger cells $\mathrm{Fe}_{64}, \mathrm{Mg}_{30} \mathrm{Fe}_{30} \mathrm{O}_{30}, \mathrm{Mg}_{45} \mathrm{Fe}_{45} \mathrm{O}_{45} \mathrm{Mg}_{40} \mathrm{O}_{40}$, and $\mathrm{Mg}_{50} \mathrm{O}_{50}$.

\footnotetext{
*Corresponding author

Email address: swahl@berkeley.edu (Sean M.Wahl )
} 
Table 1. Thermodynamic functions derived from DFT-MD simulations.

\begin{tabular}{|c|c|c|c|c|c|c|c|}
\hline $\begin{array}{c}P \\
(\mathrm{GPa})\end{array}$ & $\begin{array}{c}T \\
(\mathrm{~K})\end{array}$ & system & $\begin{array}{c}\rho \\
\left(\mathrm{g} / \mathrm{cm}^{3}\right)\end{array}$ & $\begin{array}{c}P \\
(\mathrm{GPa})\end{array}$ & $\begin{array}{c}U \\
(\mathrm{eV})\end{array}$ & $\begin{array}{c}S \\
\left(k_{B}\right)\end{array}$ & $\begin{array}{c}G \\
(\mathrm{eV})\end{array}$ \\
\hline \multirow[t]{3}{*}{0} & 4000 & $\mathrm{Mg}_{24} \mathrm{Fe}_{24} \mathrm{O}_{24}$, liq & 2.861 & $-0.41(17)$ & $-323(1)$ & $1064(4)$ & $-689.3(1)$ \\
\hline & . & $\mathrm{Fe}_{32}$,liq & 6.885 & $0.06(21)$ & $-206.53(9)$ & $496.7(4)$ & $-377.74(3)$ \\
\hline & & $\mathrm{Mg}_{32} \mathrm{O}_{32}$, liq & 2.003 & $0.60(14)$ & $-275.0(5)$ & $776(2)$ & $-542.40(5)$ \\
\hline \multirow[t]{3}{*}{0} & 5000 & $\mathrm{Mg}_{24} \mathrm{Fe}_{24} \mathrm{O}_{24}$, liq & 2.556 & $0.15(16)$ & $-289(1)$ & $1152(3)$ & $-785.4(2)$ \\
\hline & . & $\mathrm{Fe}_{32}$,liq & 6.340 & $-0.05(34)$ & $-189.8(1)$ & $540.1(4)$ & $-422.52(2)$ \\
\hline & & $\mathrm{Mg}_{32} \mathrm{O}_{32}$, liq & 1.660 & $0.10(10)$ & $-242.8(8)$ & $860(2)$ & $-613.17(6)$ \\
\hline \multirow[t]{3}{*}{0} & 6000 & $\mathrm{Mg}_{24} \mathrm{Fe}_{24} \mathrm{O}_{24}$, liq & 2.195 & $0.639(88)$ & $-251.9(8)$ & $1231(2)$ & $-888.3(1)$ \\
\hline & . & $\mathrm{Fe}_{32}$,liq & 5.740 & $-0.02(12)$ & $-170.8(1)$ & $580.2(3)$ & $-470.80(3)$ \\
\hline & & $\mathrm{Mg}_{32} \mathrm{O}_{32}$, liq & 1.435 & $0.707(49)$ & $-213(1)$ & $924(2)$ & $-690.65(9)$ \\
\hline \multirow{12}{*}{$\begin{array}{c}50 \\
.\end{array}$} & 5000 & $\mathrm{Mg}_{24} \mathrm{Fe}_{24} \mathrm{O}_{24}$, liq & 5.237 & $49.28(40)$ & $-315.5(7)$ & $963(2)$ & $-502.0(2)$ \\
\hline & . & $\mathrm{Fe}_{32}$,liq & 8.805 & $51.36(34)$ & $-203.3(3)$ & $475.0(6)$ & $-302.73(2)$ \\
\hline & . & $\mathrm{Mg}_{32} \mathrm{O}_{32}, \mathrm{liq}$ & 3.582 & $51.41(26)$ & $-261.4(5)$ & $703(1)$ & $-377.59(9)$ \\
\hline & . & $\mathrm{Mg}_{6} \mathrm{Fe}_{54} \mathrm{O}_{6}$, liq & 7.797 & $50.61(43)$ & $-370.3(5)$ & $988(1)$ & $-579.56(9)$ \\
\hline & . & $\mathrm{Mg}_{16} \mathrm{Fe}_{38} \mathrm{O}_{16}$, liq & 6.347 & $49.70(24)$ & $-338.0(8)$ & $995(2)$ & $-540.61(8)$ \\
\hline & . & $\mathrm{Mg}_{30} \mathrm{Fe}_{13} \mathrm{O}_{30}$,liq & 4.461 & $49.73(22)$ & $-302.3(7)$ & $911(2)$ & $-470.07(8)$ \\
\hline & . & $\mathrm{Mg}_{36} \mathrm{Fe}_{4} \mathrm{O}_{36}$, liq & 3.838 & $47.6(12)$ & $-317(3)$ & $855(8)$ & $-459.4(1)$ \\
\hline & . & $\mathrm{Mg}_{30} \mathrm{Fe}_{30} \mathrm{O}_{30}$, liq & 5.237 & $47.94(21)$ & $-400(1)$ & $1190(3)$ & $-627.26(8)$ \\
\hline & . & $\mathrm{Mg}_{48} \mathrm{Fe}_{48} \mathrm{O}_{48}$, liq & 5.237 & $48.49(21)$ & $-640(1)$ & $1908(3)$ & $-1004.9(2)$ \\
\hline & . & $\mathrm{Fe}_{64}, \mathrm{liq}$ & 8.805 & $50.57(18)$ & $-407.6(3)$ & $947.6(6)$ & $-605.53(2)$ \\
\hline & . & $\mathrm{Mg}_{40} \mathrm{O}_{40}$, liq & 3.582 & $40.2(11)$ & $-365(3)$ & $788(8)$ & $-470.9(2)$ \\
\hline & . & $\mathrm{Mg}_{50} \mathrm{O}_{50}$, liq & 3.582 & $44.43(37)$ & $-438(2)$ & $1023(4)$ & $-587.2(2)$ \\
\hline \multirow[t]{3}{*}{50} & 6000 & $\mathrm{Mg}_{24} \mathrm{Fe}_{24} \mathrm{O}_{24}$, liq & 5.083 & $49.36(25)$ & $-291.5(7)$ & $1027(2)$ & $-587.3(1)$ \\
\hline & . & $\mathrm{Fe}_{32}$,liq & 8.537 & $50.95(21)$ & $-191.6(1)$ & $506.5(3)$ & $-345.03(2)$ \\
\hline & & $\mathrm{Mg}_{32} \mathrm{O}_{32}$, liq & 3.446 & $49.50(24)$ & $-243.1(5)$ & $754(1)$ & $-439.09(4)$ \\
\hline \multirow[t]{12}{*}{50} & 7500 & $\mathrm{Mg}_{24} \mathrm{Fe}_{24} \mathrm{O}_{24}$, liq & 4.868 & $50.09(41)$ & $-254(2)$ & $1110(3)$ & $-725.7(1)$ \\
\hline & . & $\mathrm{Fe}_{32}$,liq & 8.164 & $50.59(52)$ & $-173.9(3)$ & $545.9(5)$ & $-413.29(2)$ \\
\hline & . & $\mathrm{Mg}_{32} \mathrm{O}_{32}, \mathrm{liq}$ & 3.301 & $51.09(60)$ & $-212(1)$ & $823(2)$ & $-540.85(6)$ \\
\hline & . & $\mathrm{Mg}_{6} \mathrm{Fe}_{54} \mathrm{O}_{6}$,liq & 7.221 & $49.04(17)$ & $-317.2(3)$ & $1119.5(5)$ & $-806.98(5)$ \\
\hline & . & $\mathrm{Mg}_{16} \mathrm{Fe}_{38} \mathrm{O}_{16}$, liq & 5.867 & $49.54(29)$ & $-278.9(8)$ & $1142(1)$ & $-772.40(6)$ \\
\hline & . & $\mathrm{Mg}_{30} \mathrm{Fe}_{13} \mathrm{O}_{30}$,liq & 4.115 & $49.90(27)$ & $-243.4(7)$ & $1058(1)$ & $-683.67(8)$ \\
\hline & . & $\mathrm{Mg}_{36} \mathrm{Fe}_{4} \mathrm{O}_{36}$, liq & 3.538 & $50.10(17)$ & $-250.7(7)$ & $1020(1)$ & $-664.90(8)$ \\
\hline & . & $\mathrm{Mg}_{30} \mathrm{Fe}_{30} \mathrm{O}_{30}$,liq & 4.868 & $49.80(16)$ & $-321(1)$ & $1382(2)$ & $-907.77(9)$ \\
\hline & . & $\mathrm{Mg}_{48} \mathrm{Fe}_{48} \mathrm{O}_{48}$, liq & 4.868 & $50.13(15)$ & $-507(1)$ & $2222(2)$ & $-1451.7(2)$ \\
\hline & . & $\mathrm{Fe}_{64}$,liq & 8.164 & $50.62(24)$ & $-347.7(3)$ & $1090.7(5)$ & $-825.75(2)$ \\
\hline & . & $\mathrm{Mg}_{40} \mathrm{O}_{40}$, liq & 3.301 & $50.75(23)$ & $-264.9(7)$ & $1026(1)$ & $-674.98(4)$ \\
\hline & . & $\mathrm{Mg}_{50} \mathrm{O}_{50}$, liq & 3.301 & $51.40(27)$ & $-330(1)$ & $1284(2)$ & $-843.19(7)$ \\
\hline
\end{tabular}


Table 1. (cont'd)

\begin{tabular}{|c|c|c|c|c|c|c|c|}
\hline $\begin{array}{c}P \\
(\mathrm{GPa})\end{array}$ & $\begin{array}{c}T \\
(\mathrm{~K})\end{array}$ & system & $\begin{array}{c}\rho \\
\left(\mathrm{g} / \mathrm{cm}^{3}\right)\end{array}$ & $\begin{array}{c}P \\
(\mathrm{GPa})\end{array}$ & $\begin{array}{c}U \\
(\mathrm{eV})\end{array}$ & $\begin{array}{c}S \\
\left(k_{B}\right)\end{array}$ & $\begin{array}{c}G \\
(\mathrm{eV})\end{array}$ \\
\hline 50 & 10000 & $\mathrm{Mg}_{24} \mathrm{Fe}_{24} \mathrm{O}_{24}$,liq & 4.546 & $51.31(32)$ & $-197(1)$ & $1210(2)$ & $-976.4(1)$ \\
\hline . & . & $\mathrm{Fe}_{32}$,liq & 7.536 & $49.92(47)$ & $-142.0(2)$ & $601.3(3)$ & $-537.28(3)$ \\
\hline . & & $\mathrm{Mg}_{32} \mathrm{O}_{32}$,liq & 3.054 & $50.64(51)$ & $-163(1)$ & $909(2)$ & $-727.8(1)$ \\
\hline \multirow{3}{*}{100} & 6000 & $\mathrm{Mg}_{24} \mathrm{Fe}_{24} \mathrm{O}_{24}$, liq & 6.144 & $100.54(60)$ & $-264(1)$ & $969(3)$ & $-375.5(1)$ \\
\hline & . & $\mathrm{Fe}_{32}$,liq & 9.771 & $97.45(69)$ & $-188.0(5)$ & $476(1)$ & $-244.43(4)$ \\
\hline & & $\mathrm{Mg}_{32} \mathrm{O}_{32}$, sol & 4.432 & $100.04(18)$ & $-261.7(3)$ & $598.8(7)$ & $-269.70(8)$ \\
\hline \multirow[t]{3}{*}{100} & 6700 & $\mathrm{Mg}_{24} \mathrm{Fe}_{24} \mathrm{O}_{24}$,liq & 6.042 & $98.34(38)$ & $-254.2(7)$ & $998(1)$ & $-434.5(1)$ \\
\hline & & $\mathrm{Fe}_{32}$,liq & 9.660 & $99.69(82)$ & $-179.6(5)$ & $495(1)$ & $-273.67(4)$ \\
\hline & & $\mathrm{Mg}_{32} \mathrm{O}_{32}, \mathrm{sol}$ & 4.381 & $100.46(35)$ & $-250.5(6)$ & $627(1)$ & $-307.19(8)$ \\
\hline \multirow[t]{3}{*}{100} & 7000 & $\mathrm{Mg}_{24} \mathrm{Fe}_{24} \mathrm{O}_{24}$, liq & 6.027 & $101.60(35)$ & $-238.4(8)$ & $1027(1)$ & $-461.2(1)$ \\
\hline & . & $\mathrm{Fe}_{32}$,liq & 9.611 & $100.24(30)$ & $-176.2(2)$ & $502.5(3)$ & $-286.64(2)$ \\
\hline & & $\mathrm{Mg}_{32} \mathrm{O}_{32}$, sol & 4.360 & $100.53(42)$ & $-245.4(3)$ & $637.3(6)$ & $-323.25(7)$ \\
\hline \multirow[t]{3}{*}{100} & 8000 & $\mathrm{Mg}_{24} \mathrm{Fe}_{24} \mathrm{O}_{24}$, liq & 5.932 & $103.41(68)$ & $-218(1)$ & $1068(2)$ & $-551.3(2)$ \\
\hline & & $\mathrm{Fe}_{32}$,liq & 9.388 & $99.43(71)$ & $-165.0(5)$ & $527.1(8)$ & $-331.12(4)$ \\
\hline & & $\mathrm{Mg}_{32} \mathrm{O}_{32}$, liq & 4.034 & $100.57(67)$ & $-183.8(7)$ & $785(1)$ & $-393.43(7)$ \\
\hline \multirow[t]{3}{*}{100} & 10000 & $\mathrm{Mg}_{24} \mathrm{Fe}_{24} \mathrm{O}_{24}$,liq & 5.664 & $100.86(98)$ & $-176(2)$ & $1147(2)$ & $-742.5(2)$ \\
\hline & 年 & $\mathrm{Fe}_{32}$,liq & 8.998 & $98.79(49)$ & $-142.5(4)$ & $567.2(4)$ & $-425.41(2)$ \\
\hline & & $\mathrm{Mg}_{32} \mathrm{O}_{32}$,liq & 3.842 & $99.23(52)$ & $-145(2)$ & $856(2)$ & $-534.78(8)$ \\
\hline \multirow[t]{2}{*}{100} & 15000 & $\mathrm{Mg}_{24} \mathrm{Fe}_{24} \mathrm{O}_{24}$, liq & 5.157 & $101.17(74)$ & $-75(2)$ & $1281(2)$ & $-1267.13(10)$ \\
\hline & & $\mathrm{Fe}_{32}$,liq & 8.145 & $102.48(80)$ & $-81.2(5)$ & $645.5(4)$ & $-688.15(2)$ \\
\hline \multirow[t]{3}{*}{400} & 8000 & $\mathrm{Mg}_{24} \mathrm{Fe}_{24} \mathrm{O}_{24}$, liq & 9.117 & $398.69(56)$ & $-24(1)$ & $921(2)$ & $390.9(2)$ \\
\hline & 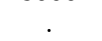 & $\mathrm{Fe}_{32}$,liq & 13.469 & $397.7(10)$ & $-93.1(8)$ & $453(2)$ & $145.0(2)$ \\
\hline & & $\mathrm{Mg}_{32} \mathrm{O}_{32}$, sol & 6.487 & $399.95(25)$ & $-69.8(3)$ & $580.4(5)$ & $354.3(1)$ \\
\hline \multirow[t]{3}{*}{400} & 9300 & $\mathrm{Mg}_{24} \mathrm{Fe}_{24} \mathrm{O}_{24}$, liq & 9.010 & $400.51(49)$ & $5(2)$ & $974(2)$ & $285.7(2)$ \\
\hline & & $\mathrm{Fe}_{32}$,liq & 13.583 & $398.04(53)$ & $-95.6(5)$ & $445.8(7)$ & $92.59(8)$ \\
\hline & & $\mathrm{Mg}_{32} \mathrm{O}_{32}, \mathrm{sol}$ & 6.434 & $400.24(54)$ & $-51.3(5)$ & $615.1(8)$ & $286.8(1)$ \\
\hline \multirow[t]{3}{*}{400} & 10000 & $\mathrm{Mg}_{24} \mathrm{Fe}_{24} \mathrm{O}_{24}$, liq & 8.954 & $399.46(67)$ & $17(2)$ & $998(2)$ & $225.5(2)$ \\
\hline & & $\mathrm{Fe}_{32}$,liq & 13.263 & $397.6(25)$ & $-75(2)$ & $485(2)$ & $65.3(1)$ \\
\hline & & $\mathrm{Mg}_{32} \mathrm{O}_{32}, \mathrm{sol}$ & 6.393 & $397.31(43)$ & $-41.8(3)$ & $632.4(5)$ & $249.7(1)$ \\
\hline 400 & 12500 & $\mathrm{Mg}_{24} \mathrm{Fe}_{24} \mathrm{O}_{24}$, liq & 8.753 & $399.86(65)$ & $63(1)$ & 1071(1) & $2.5(2)$ \\
\hline \multirow{2}{*}{400} & the & $\mathrm{Fe}_{32}$,liq & 12.938 & $400.3(12)$ & $-46.0(9)$ & $530.1(9)$ & $-44.36(5)$ \\
\hline & & $\mathrm{Mg}_{32} \mathrm{O}_{32}$, liq & 6.100 & $396.44(75)$ & $61(1)$ & $794(1)$ & $82.3(1)$ \\
\hline 400 & 15000 & $\mathrm{Mg}_{24} \mathrm{Fe}_{24} \mathrm{O}_{24}$,liq & 8.553 & $399.7(11)$ & $117(2)$ & $1138(2)$ & $-235.9(1)$ \\
\hline . & (2000 & $\mathrm{Fe}_{32}$,liq & 12.688 & $403.62(99)$ & $-19.3(7)$ & $562.1(6)$ & $-161.93(3)$ \\
\hline . & & $\mathrm{Mg}_{32} \mathrm{O}_{32}$,liq & 5.943 & $396.6(11)$ & $114(2)$ & $858(2)$ & $-95.8(1)$ \\
\hline
\end{tabular}




\subsection{Saturation limits}

$\Delta G_{m i x}$ can be related to the volume change associated with the insertion of an iron of atom into hydrogen, as other contributions are constant with respect concentration. It can be shown that results for simulations with a 1:n solute ratio can be generalized to a ratio of $1: m$ using

$$
\begin{aligned}
\Delta G_{c} \approx & F_{0}\left(F e_{m} M g O\right)-F_{0}\left(F e_{m}\right)-F_{0}(M g O) \\
& -\left[F F_{0}\left(F e_{n} M g O\right)-F_{0}\left(F e_{n}\right)-F_{0}(M g O)\right] \\
= & -k_{B} T \log \left\{\frac{\left[V\left(\mathrm{Fe}_{n} \mathrm{MgO}\right)+\frac{m-n}{n} V\left(\mathrm{Fe}_{n}\right)\right]^{m+1}\left[V\left(\mathrm{Fe}_{n}\right)\right]^{n}}{\left[V\left(\mathrm{Fe}_{n}\right) \frac{m}{n}\right]^{m}\left[V\left(\mathrm{Fe}_{n} \mathrm{MgO}\right)\right]^{n+1}}\right\},
\end{aligned}
$$

where $\Delta G_{c}=\Delta G_{m i x}(1: m)-\Delta G_{m i x}(1: n)$, and $V\left(\mathrm{Fe}_{n}\right)$ and $V\left(\mathrm{Fe}_{n} \mathrm{MgO}\right)$ are the volumes for the simulations of hydrogen and the solution respectively. This allows us to approximate the saturation limit for $\mathrm{MgO}$ in Fe based only on $\Delta G_{m i x}$ and $V$ of our lowest concentration simulation, and $V$ of both of the endmember compositions. We note that this low-concentration limit assumes that the self interaction of the $\mathrm{MgO}$ 'solute' is negligible in our lowest concentration, $\mathrm{Mg}_{6} \mathrm{Fe}_{54} \mathrm{O}_{6}$. While this is not exact, we present it as a estimate for extrapolating these results to low $\mathrm{MgO}$ concentrations. In doing so, we demonstrate that these calculations are consistent with $\mathrm{Mg}$ concentrations the below detection limit of laser-heated diamond anvil cell experiments performed at lower temperatures.

\subsection{Comparison of thermodynamic integration with different classical poten- tials}

The classical pair potentials are derived by fitting the forces and positions along a pre-computed DFT-MD trajectory. The potentials are constructed to approach zero for large separations. For small separations where the trajectories provide no information, linear extrapolation is used, which means our pair potentials are finite at the origin. All of the results presented in the paper used this fitting procedure. Figure 1 shows an example for the pair potentials for liquid $\mathrm{MgO}$ and $50 \mathrm{GPa}$ and $6000 \mathrm{~K}$. While the $\mathrm{Mg}-\mathrm{Mg}$ and $\mathrm{O}-\mathrm{O}$ potentials are purely repulsive, the deep mininum in the $\mathrm{Mg}-\mathrm{O}$ potential represents the attractive forces between ions of opposite charge.

Table 2 provide all terms of the thermodynamic integration procedure. In order to test how robust our approach is, we constructed a different set of pair potential where we eliminated all bonding forces. The values of these 
Table 2. Comparison of integration paths using different classical potentials.

\begin{tabular}{cccccc}
\hline \hline \multicolumn{1}{c}{ Potentials } & \multicolumn{1}{c}{$F_{\text {an }}$} & $F_{\text {an } \rightarrow \mathrm{cl}}$ & \multicolumn{1}{c}{$F_{\mathrm{cl}}$} & \multicolumn{1}{c}{$F_{\mathrm{cl} \rightarrow \mathrm{DFT}}$} & $F_{\mathrm{DFT}}$ \\
\hline Regular, bonding potentials & -427.151 & 27.962 & -399.189 & $-233.825 \pm 0.031$ & $-633.014 \pm 0.031$ \\
Non-bonding potentials & -427.151 & 204.129 & -223.022 & $-409.927 \pm 0.080$ & $-632.950 \pm 0.080$ \\
\hline
\end{tabular}

non-bonding potentials are constrained to be positive, asymptoting to zero without a miniumum. Obviously they are a poor representation of the DFT forces in the system and therefore the free energy differences between the DFT and the classical system, $F_{\mathrm{cl} \rightarrow \text { DFT }}$, given in table 2 , is much larger than for our regular potentials. However, when the values for $F_{a n}, F_{a n \rightarrow c l}$, and $F_{\mathrm{cl} \rightarrow \text { DFT }}$ are added, we recover the results for $F_{D F T}$ within the $1 \sigma$ error bars. This demonstrates that out free energy calculations are not sensitive to the details how we construct our classical potentials.

Although the correct final result is found when using an unrealistic potential, the efficiency for the thermodynamic integration is the highest when the classical forces best match the DFT forces. Figure 2 shows the calculated values of $\left\langle V_{D F T}-V_{\mathrm{cl}}\right\rangle$ as a function of $\lambda$ using the regular "bonding" potential, and for the "non-bonding potential". Here each plotted value of lambda represents and independent DFT-md simulation with using that fraction of DFT forces, along with the complementary fraction of classical forces. The integral of this function give the helmholtz free energy $F_{\mathrm{cl} \rightarrow \mathrm{DFT}}$. In the first case, the function $\left\langle V_{D F T}-V_{\mathrm{cl}}\right\rangle$ in figure 2 depends weakly on $\lambda$. The function is almost linear and the differnce between values at $\lambda=0$ and 1 is small. When both criteria are satisfied, very few $\lambda$ points are needed to evaluate the integral. The simulations with non-bonding potentials experience larger fluctuations due to the greater mismatch in forces, leading to a larger statistical uncertainty. As a result, these simulations required longer simulation times to match the results found with bonding potentials.

Figure 3 shows $\left\langle V_{\mathrm{cl}}\right\rangle$ as a function of $\lambda$ from classical monte carlo simulations. The integration of $F_{a n \rightarrow c l}$ function becomes strongly non-linear as $\lambda$ approaches zero, the non-interacting case. Since classical simulations are approximately $10^{5}$ times more efficient, it is possible to obtain very close sampling of the a cusp in the integrated function. 
Using the definition for the ensemble averaged potential at a given $\lambda$

$$
\left\langle V_{\mathrm{cl}}\right\rangle_{\lambda}=\frac{\int d \mathbf{r} V_{\mathrm{cl}}(\mathbf{r}) e^{-\beta \lambda V_{\mathrm{cl}}(\mathbf{r})}}{Z},
$$

where $Z$ is the partition function

$$
Z=\int d \mathbf{r} e^{-\beta \lambda V_{\mathrm{cl}}(\mathbf{r})},
$$

we find the following expression as $\lambda \rightarrow 0$

$$
\begin{aligned}
\left\langle V_{\mathrm{cl}}\right\rangle_{\lambda \rightarrow 0} & =\frac{\int d \mathbf{r} V_{\mathrm{cl}}}{\int d \mathbf{r} 1} \\
& =\frac{1}{V} \int d r r^{2} V_{\mathrm{cl}}(r)
\end{aligned}
$$

Then from the deriviative of $\left\langle V_{\mathrm{cl}}\right\rangle_{\lambda}$ in equation 2 and 4

$$
\begin{aligned}
\frac{d\left\langle V_{\mathrm{cl}}\right\rangle}{d \lambda} & =\frac{1}{Z^{2}}\left[Z \int d \mathbf{r}(-\beta) V_{\mathrm{cl}}^{2}(\mathbf{r}) e^{-\beta \lambda V_{\mathrm{cl}}(\mathbf{r})}-(-\beta)\left\{\int d \mathbf{r} V_{\mathrm{cl}}(\mathbf{r}) e^{-\beta \lambda V_{\mathrm{cl}}(\mathbf{r})}\right\}^{2}\right] \\
& =(-\beta)\left[\left\langle V_{\mathrm{cl}}^{2}\right\rangle-\left\langle V_{\mathrm{cl}}\right\rangle^{2}\right] \\
\left.\frac{d\left\langle V_{\mathrm{cl}}\right\rangle}{d \lambda}\right|_{\lambda \rightarrow 0} & =(-\beta)\left[\frac{1}{V} \int d r r^{2} V_{\mathrm{cl}}^{2}(r)-\left\{\frac{1}{V} \int d r r^{2} V_{\mathrm{cl}}(r)\right\}^{2}\right]
\end{aligned}
$$

This give us the slope and intercept for the integration at $\lambda=0$, necessary to correctly integrate the cusp. Becauase of the extreme difference in computational efficiency, it is always best to adjust the classical potential to match the DFT forces.

\subsection{Verification of thermodynamic integration in multicomponent systems}

The second test is to verify that, in a multi-component system, the integration path does not effect the results. An integration path needs to be constructed that connects a system with $\mathrm{Mg}-\mathrm{Mg}, \mathrm{Mg}-\mathrm{O}$, and O-O pair potentials with an non-interacting system. In our standard proceduce we turn on all pair potentials simultaneously by changing $\lambda_{1}=\lambda_{2}=\lambda_{3}$ from 0 to 1 .

$$
V_{\lambda_{1} \lambda_{2} \lambda_{3}}=\lambda_{1} V_{\mathrm{Mg}-\mathrm{Mg}}+\lambda_{2} V_{\mathrm{Mg}-\mathrm{O}}+\lambda_{3} V_{\mathrm{O}-\mathrm{O}}
$$


Table 3. Comparison of different integration paths using classical potentials.

\begin{tabular}{cclc}
\hline \hline Potential & $F_{\text {an }}$ & \multicolumn{1}{c}{$F_{\text {step }}$} & $F_{\text {an }}+\sum F_{\text {step }}$ \\
\hline Non-bonding & -427.151 & $F_{000 \rightarrow 111}=204.196(27)$ & $-222.955(27)$ \\
\hline Non-bonding & -427.151 & $F_{000 \rightarrow 010}=16.328(6)$ & $-222.953(45)$ \\
& & $F_{010 \rightarrow 111}=187.870(39)$ & \\
\hline Non-bonding & -427.151 & $F_{000 \rightarrow 101}=166.363(15)$ & $-222.962(21)$ \\
& & $F_{101 \rightarrow 111}=37.826(18)$ & \\
\hline Non-bonding & -427.151 & $F_{000 \rightarrow 100}=104.323(11)$ & $-222.954(52)$ \\
& & $F_{100 \rightarrow 110}=31.225(10)$ & \\
\hline Regular & -427.151 & $F_{110 \rightarrow 111}=68.650(31)$ & \\
\hline Regular & -427.151 & $F_{000 \rightarrow 111}=28.028(31)$ & $-399.123(31)$ \\
\hline & & $F_{101 \rightarrow 101}=182.820(16)$ & $-399.123(37)$ \\
& & & \\
\hline
\end{tabular}

However, as we will now demonstrate, alternative integration paths will give the same results. We compare different integration paths to calculate the free energy of the classical system, $F_{\mathrm{cl}}$, for both the regular and non-bonding potentials. This is comparison can not be made directly for $F_{\mathrm{cl} \rightarrow \text { DFT }}$ because tracking the intereaction of different species separarately is not possible in a Kohn-Sham formulation.

In line 2 of table 3, we turn on the $\mathrm{Mg}-\mathrm{O}$ potential in the first integration step $\left(F_{000 \rightarrow 010}\right)$ and then switch on the $\mathrm{Mg}-\mathrm{Mg}$ and $\mathrm{O}-\mathrm{O}$ potentials in the second and final integration step $\left(F_{101 \rightarrow 111}\right)$. The indices refer to the three $\lambda$ values for $\mathrm{Mg}-\mathrm{Mg}, \mathrm{Mg}-\mathrm{O}$, and $\mathrm{O}-\mathrm{O}$ potentials, respectively. In line 3 of table 3 , we interchange both integration steps. In line 4 , we performed three integration steps turning on the one potential after the other. In the last column we compare the classical free energies after adding the results from every integration step to $F_{\text {an }}$. The results agree within the statistical uncertainties demonstrating that the same classical free energies can be obtained for four different integration paths using non-bonding potentials.

In table 3, we also show the results for two integration paths using regular, bonding potentials. We find consistent results when we either turn on all potentials simultaneously and when we switch on the $\mathrm{Mg}-\mathrm{Mg}$ and $\mathrm{O}-\mathrm{O}$ potentials in the first step and the $\mathrm{Mg}-\mathrm{O}$ potential in the second. We were not able, however, to turn on the attractive $\mathrm{Mg}-\mathrm{O}$ alone because the system 
becomes unstable due to the imbalance between attractive and missing repulsive forces. This is similar to what happens in the case of a first-order phase transition, which over which thermodynamic integrations are also invalid. Nevertheless, this test demonstrates that different integration paths give consistent results also for the systems with attractive forces when care is taken to taken to avoid instabilities.
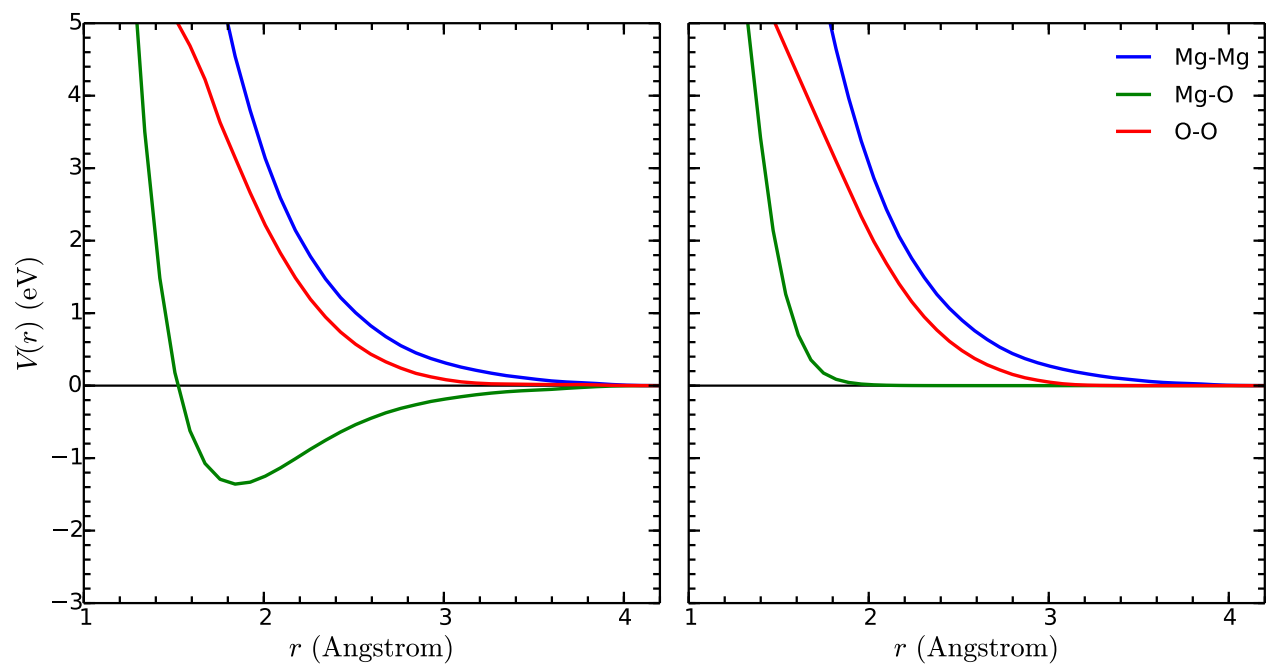

Figure 1. Example pair potentials for liquid $\mathrm{MgO}$ at $50 \mathrm{GPa}$ and $6000 \mathrm{~K}$. Left: Regular pair potentials fit to DFT-MD simulations, with a linear extrapolation at small separation and an asymptote to 0 at large separation. All of the results presented in the paper used this fitting procedure. Right: Non-bonding potential fit with the same procedure, but constraining values to be positive. Included for comparison with the pair potentials in table 2 . 


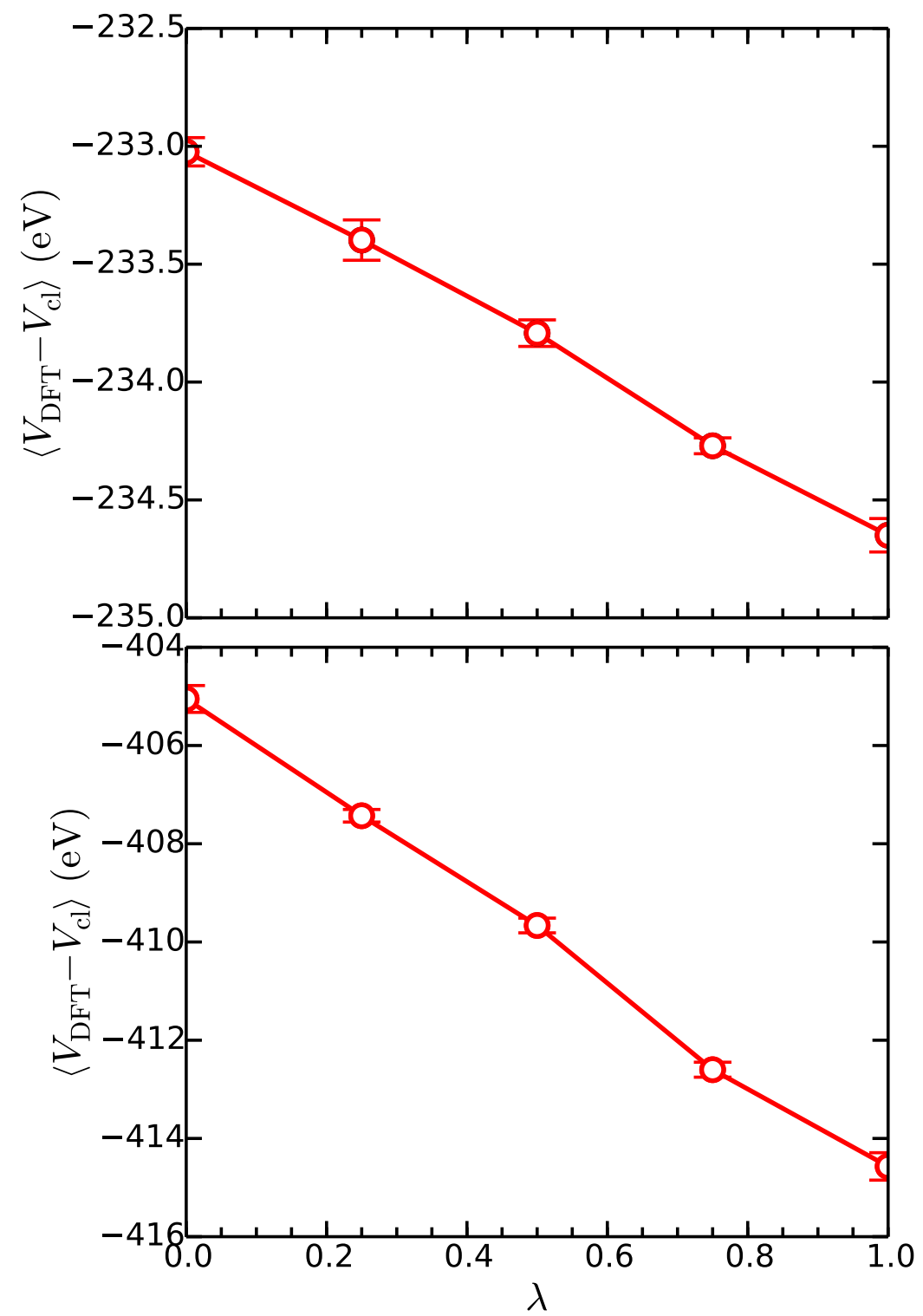

Figure 2. The integration path to find $F_{c l \rightarrow D F T}$ potentials for $\mathrm{MgO}$ at 50 $\mathrm{GPa} 6000 \mathrm{~K}$, using the regular, bonding pair potentials (upper) and the non-bonding pair potentials (lower). 


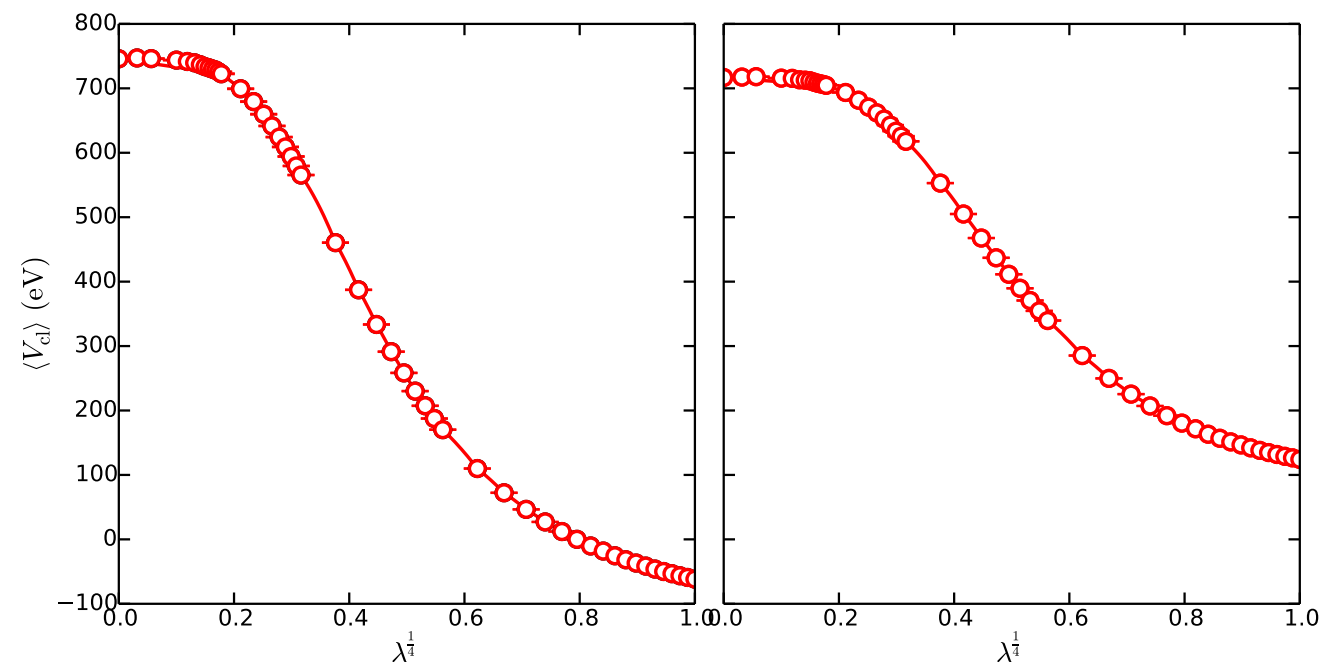

Figure 3. The integration path to find $F_{a n \rightarrow c l}$ potentials for $\mathrm{MgO}$ at 50 GPa $6000 \mathrm{~K}$, using the regular, bonding pair potentials (left) and the non-bonding pair potentials (right). These are plotted against the integration parameter, $\lambda$, to the $1 / 4$ power. 